\title{
Using Indistinguishability Obfuscation via UCEs
}

\author{
Christina Brzuska ${ }^{1} \quad$ Arno Mittelbach ${ }^{2}$ \\ ${ }^{1}$ Tel Aviv University, Israel \\ ${ }^{2}$ Darmstadt University of Technology, Germany \\ christina.brzuska@gmail.com mail@arno-mittelbach.de
}

\begin{abstract}
We provide the first standard model construction for a powerful class of Universal Computational Extractors (UCEs; Bellare et al. Crypto 2013) based on indistinguishability obfuscation. Our construction suffices to instantiate $q$-query correlation-secure hash functions and to extract polynomially many hardcore bits from any one-way function.

For many cryptographic primitives and in particular for correlation-secure hash functions all known constructions are in the random-oracle model. Indeed, recent negative results by Wichs (ITCS 2013) rule out a large class of techniques to prove the security of correlation-secure hash functions in the standard model. Our construction is based on puncturable PRFs (Sahai und Waters; STOC 2014) and indistinguishability obfuscation. However, our proof also relies on point obfuscation under auxiliary inputs (AIPO). This is crucial in light of Wichs' impossibility result. Namely, Wichs proves that it is often hard to reduce two-stage games (such as UCEs) to a "one-stage assumption" such as DDH. In contrast, AIPOs and their underlying assumptions are inherently two-stage and, thus, allow us to circumvent Wichs' impossibility result.

Our positive result is also noteworthy insofar as Brzuska, Farshim and Mittelbach (Crypto 2014) have shown recently, that $\mathrm{iO}$ and some variants of UCEs are mutually exclusive. Our results, hence, validate some of the new UCE notions that emerged as a response to the iO-attack.
\end{abstract}

Keywords. Correlation-secure hash functions, hardcore functions, indistinguishability obfuscation, differing-inputs obfuscation, point-function obfuscation, auxiliary-input obfuscation, universal computational extractors (UCEs) 


\section{Contents}

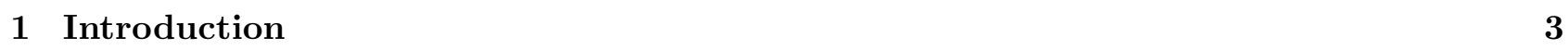

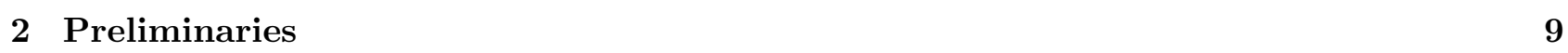

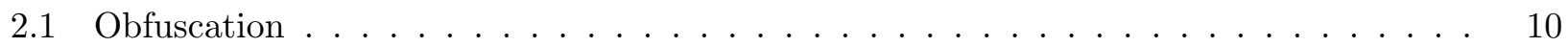

2.2 Universal Computational Extractors (UCE) . . . . . . . . . . . . . . . . . . . 13

2.3 Puncturable PRFs $\ldots \ldots \ldots \ldots \ldots \ldots \ldots \ldots \ldots \ldots$

3 UCEs from iO and Point Obfuscation $\quad 15$

3.1 Strongly Unpredictable and $q$-Query Sources . . . . . . . . . . . . . . . . . . . . 16

$3.2 \quad$ A UCE Construction Secure Against Sources in $\mathcal{S}^{\text {s-cup }} \cap \mathcal{S}^{1 \text {-query }} \ldots \ldots \ldots$

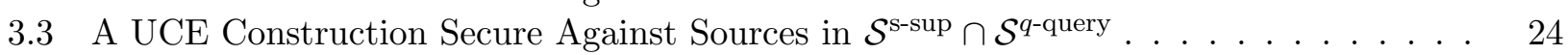

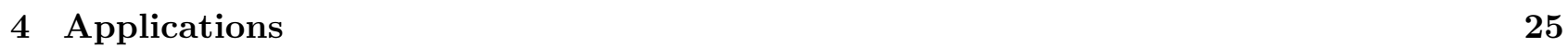

4.1 Hash Functions Secure under Correlated Inputs . . . . . . . . . . . . . . . . . . . . . 25

4.2 Universal Hardcore Functions $\ldots \ldots \ldots \ldots$. . . . . . . . . . . . . . . . . 26

\begin{tabular}{|lll}
\hline 5 & The BFM Impossibility Result & 27
\end{tabular}

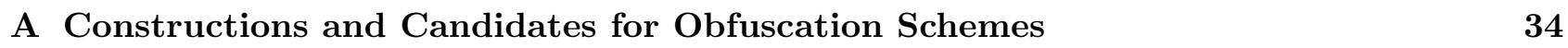

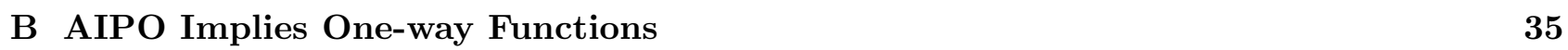

\begin{tabular}{|l|l|l|}
\hline C Proof of Theorem & 3.5 & 35
\end{tabular} 


\section{Introduction}

For many cryptographic primitives, it is easy to construct a secure scheme in the random oracle model, but it is hard to give a construction in the standard model. For example, correlated-input hash functions (CIH) which were introduced by Goyal, O'Neill, and Rao [GOR11, are easy to construct in the random oracle model, because the random oracle itself is secure under correlated inputs. However, up to now, no standard-model construction is known, and indeed, a recent black-box separation by Wichs [Wic13] explains why it is so hard to construct them. Namely, the security definition of a CIH involves a pair of adversaries $\left(\mathcal{A}_{1}, \mathcal{A}_{2}\right)$ and is thus a two-stage game (i.e., the adversary is not a single algorithm but consists of two separate algorithms). The first adversary samples correlated inputs $\left(x_{1}, \ldots, x_{t}\right)$. Then a hash key $\mathrm{hk}$ is generated and the second adversary with access to hk needs to distinguish between getting a tuple of random strings and getting the tuple $\left(H\left(\mathrm{hk}, x_{1}\right), \ldots, H\left(\mathrm{hk}, x_{t}\right)\right)$. Now, Wichs employs a meta reduction to show that it is unlikely to have a black-box reduction $\mathcal{R}$ from CIH to a (one-stage) cryptographic assumption such as the decisional Diffie-Hellman assumption (DDH). Namely, he shows that if such a reduction to DDH exists, then the DDH assumption is wrong. In his proof, he substantially exploits that the CIH game is a two-stage game. For a black-box reduction $\mathcal{R}$ it must hold that if the reduction $\mathcal{R}$ gets access to a pair of oracles $\left(\mathcal{A}_{1}, \mathcal{A}_{2}\right)$ that break $\mathrm{CIH}$, then $\mathcal{R}^{\mathcal{A}_{1}, \mathcal{A}_{2}}$ must also break DDH. Wichs constructs a pair of inefficient adversaries $\left(\mathcal{A}_{1}, \mathcal{A}_{2}\right)$ which, however, can be efficiently emulated using a stateful simulator Sim. That is, the simulator simulates both adversaries together while sharing state between them. As the reduction cannot distinguish between the two settings $\mathcal{R}^{\mathcal{A}_{1}, \mathcal{A}_{2}}$ and $\mathcal{R}^{\mathrm{Sim}}$ this breaks DDH, and hence, if we believe that DDH is a hard problem, then such an $\mathcal{R}$ cannot exist. Note that Wichs' proof is not specific to DDH, but rather applies to any one-stage assumption and presents a substantial barrier to prove security. Moreover, Wichs' impossibility result extends to a range of security notions that are specified by two-stage games.

In this paper, we use cryptographic obfuscation techniques to circumvent Wichs' impossibility result and achieve security notions that are based on two-stage assumptions. Towards this goal, the key idea is to combine point-function obfuscation and indistinguishability obfuscation.

Point And Indistinguishability obfuscation. A point function $p_{x}$ is a function that returns 1 on input $x$ and $\perp$ on all other values. A point function obfuscator under auxiliary input AIPO returns a point function $p \leftarrow \$ \operatorname{AIPO}(x)$ that hides the point $x$ even in case the adversary receives some side-channel information $z$ about $x$. More formally, the security of AIPO is defined as security for all computationally unpredictable distributions $\mathcal{D}$, that is, $\mathcal{D}$ outputs a pair $(x, z)$, where $x$ is a point and $z$ is some leakage that hides $x$ computationally. AIPO is secure, if for all computationally unpredictable $\mathcal{D},(\operatorname{AIPO}(x), z)$ is indistinguishable from $(\operatorname{AIPO}(u), z)$, where $(x, z) \leftarrow \mathcal{D}$ and $u$ is a uniformly random point. Such AIPO schemes have been constructed in [Can97, BP12].

While point function obfuscators are obfuscation schemes for a very specific class of functionalities (namely point functions) Garg et al. $\left[\mathrm{GGH}^{+} 13\right]$ have recently revived the study of general obfuscation schemes with their candidate construction of indistinguishability obfuscation. The notion of indistinguishability obfuscation is weaker than VBB-obfuscation - thereby circumventing the impossibility results of Barak et al. $\left[\mathrm{BGI}^{+} 01, \mathrm{BGI}^{+} 12\right.$ - and says intuitively that, for any two circuits that compute the same function, their obfuscations are indistinguishable. The publication of the candidate for indistinguishability obfuscation by Garg et al. inspired simultaneous breakthroughs for hard problems in various sub-areas of cryptography [SW14, BCP14, $\mathrm{ABG}^{+} 13$, GGHR14, HSW14, BZ14, BST14, GGG 14 ] including functional and deniable encryption, two-round secure multi-party computation, full-domain hash, poly-many hardcore bits from any one-way function, multi-input functional encryption and more. 
CORRElated-inPUt haSh-FUnCtions. In this paper, we give the first standard-model construction for q-query CIHs. Our CIH is not only one-way under correlated inputs, but also outputs elements that are indistinguishable from random. We will compare our notion of q-query $\mathrm{CIH}$ with other notions of CIHs shortly.

On a high-level, our construction is a de facto instantiation of a random oracle. As the behavior of a PRF is similar to that of a random function, we instantiate the random oracle by securely delegating a PRF, that is, we obfuscate a PRF with a hard coded key. Indeed, our hash-function construction only consists of a (puncturable) PRF that is obfuscated via an indistinguishability obfuscator (iO):

\section{Hash Construction: $\mathrm{iO}(\operatorname{PRF}(k,)$.$) .$}

Bellare, Stepanovs, and Tessaro (BST; [BST14]) already used this natural construction in the direct construction of hardcore functions for injective one-way functions from indistinguishability obfuscation. We will discuss BST and the relation to our our work shortly.

Noteworthy, and we will also come back to this, is that before obfuscating the PRF we need to pad the circuit to a specific length. This is needed when using indistinguishability obfuscation to move from one circuit to another one in the security proof and thus the construction must be padded to the length of the biggest circuit needed within the security proof. Jumping ahead, we note that although our construction and that of BST look identical on the outside the padding is different. For BST, the construction needs to be padded differently depending on the size of the one-way function. In turn, our padding is universal and thus, we yield a universal hardcore function that works for any one-way function.

Circumventing Wichs' impossibility Result. Although the construction is natural, proving its security is non-trivial, as the security guarantees of $\mathrm{iO}$ do not even allow us to show easily that it is hard to extract the PRF key. Towards proving the security of our construction, we build on the puncturable PRF technique by Waters and Sahai [SW14] and combine it with point function obfuscators secure under auxiliary input (AIPO).

Using AIPOs is crucial to circumvent the impossibility result by Wichs Wic13, because the security of AIPOs is defined via a two-stage security game. The first AIPO adversary samples a point, and the second adversary tries to break the obfuscation of the point function. In a sense, the impossibility result of Wichs tells us that using a two-stage assumption such as AIPO in the proof is, indeed, necessary. In particular, $\mathrm{iO}$ and PRFs are both one-stage assumptions. Note that, as AIPOs are only used in the proof and not in the construction, it might be possible that the same construction can be proven secure without making use of AIPOs possibly through some other two-stage assumption.

Universal hardcore functions for ANY ONE-WAY FUnCtion. Bellare, Stepanovs, and Tessaro (BST; BST14]) recently established that the same construction (with a different amount of padding) also yields a hardcore function for any injective one-way function, assuming a puncturable $\mathrm{PRF}$ and $\mathrm{iO}$.

For general one-way functions, BST gave a second, different construction of a hardcore function and proved it based on so-called differing-inputs obfuscation. Differing-inputs obfuscation is a stronger assumption than $\mathrm{iO}$ and has been shown conditionally impossible by Garg et al. [GGHW14a] assuming special-purpose obfuscators. Therefore, in the current version of their paper, Bellare et al. [BST14] use a weaker variant of diO that is not affected by the results of Garg et al. [GGHW14a].

In an updated version of their paper, Garg et al. GGHW14b] show that, assuming a special-purpose obfuscator and indistinguishability obfuscation for Turing Machines, there are one-way functions for which the second construction of BST cannot be a secure hardcore function, because their hardcore 
function has "output-only dependence". This means that hardcore bits $h(x)$ are completely determined by $f(x)$, or in other words, for any inputs $x$ and $x^{\prime}$ such that $f(x)=f\left(x^{\prime}\right)$ it holds that $h(x)=h\left(x^{\prime}\right)$. We note that the only candidate for $\mathrm{iO}$ for Turing machines is currently based on full diO.

The conditional negative result for output-only dependent hardcore functions does not apply to the construction $\mathrm{iO}(\operatorname{PRF}(k,)$.$) which is the construction that we use throughout this paper and which$ BST - with a different amount of padding - prove to be a hardcore function for injective one-way functions. In turn, assuming AIPO in addition to $\mathrm{iO}$ allows us to prove this construction secure for all one-way functions, even those that have many pre-images. Another difference with the BST result is that we yield a universal hardcore function for any one-way function while their padding depends on the one-way function.

Our proof builds on ideas by BST, and we will come back to their result in the context of presenting our proof techniques. We note that for our security proof, we assume AIPO in addition to iO and thereby are able to avoid diO variants altogether. The assumption of point obfuscators is currently incomparable to the assumption of differing-inputs obfuscation as well as to more restricted versions that were used by BST. It is an interesting question to explore the relationship between these assumptions.

Modularizing PROOFs VIA UCEs. We could prove the security of our construction directly, but instead, we split our proof into two parts. First, we show that our construction enjoys some useful, abstract properties. Then we use results by Bellare et al. BHK13a that show that these abstract properties suffice for the application at hand. This way, we provide a means of using $\mathrm{iO}$ in a black-box way. Our abstraction is a version of UCE security [BHK13a] that we discuss next.

The UCE Framework by Bellare, Hoang, and Keelveedhi (BHK; BHK13a]) introduces assumptions that allow us to instantiate random oracles in a wide range of applications. Loosely speaking, UCEs are PRF-like assumptions that split the distinguisher into two parts: a first adversary $\mathrm{S}$ that gets access to a keyed hash function or a random oracle (and which is called the source), and a second adversary $\mathrm{D}$ that gets the hash key hk (and which is called the distinguisher). The two algorithms together try to guess whether the source was given access to a keyed hash function (under a randomly chosen key) or to a random oracle.

Concretely, the UCE notions are defined via a two-stage UCE game (we depict the communication flow in Figure 1 and the pseudocode in Figure 2). First, the source $\mathrm{S}$ is run with oracle access to HasH (which either implements a random oracle or the hash function with a randomly chosen key hk) to output some leakage $L$. Subsequently, distinguisher D is run on the leakage $L$ and hash key hk but without access to oracle HAsH. Distinguisher D outputs a single bit $b$ indicating whether oracle HASH implements a random oracle or hash function $\mathrm{H}$ with key hk.

Without any restrictions, (S, D) can easily win the UCE game. For example, say, source $\mathrm{S}$ makes a random query $x$ to receive $y \leftarrow \operatorname{HASH}(x)$ and outputs $(x, y)$ as leakage. As distinguisher $\mathrm{D}$ knows the hash key $\mathrm{hk}$ as well as the leakage $(x, y)$, it can recompute the hash value and check whether $y=\mathrm{H}(\mathrm{hk}, x)$.

BHK present several possible restrictions on the source which give rise to various UCE notions. It turns out to be particularly useful to restrict sources to be computationally unpredictable, that is, the leakage created by the source $\mathrm{S}$ - when interacting with a random oracle - should not reveal (computationally) any of the source's queries to HASH. This notion is denoted by UCE[ $\left.\mathcal{S}^{\text {cup }}\right]$, where $\mathcal{S}^{\text {cup }}$

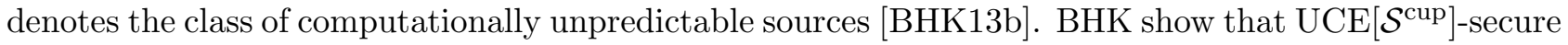
hash functions can safely replace a random oracle in a large number of interesting applications such as hardcore functions or deterministic public-key encryption [BHK13a]. In a recent work Brzuska, Farshim and Mittelbach (BFM; [BFM14]) show that UCE security with respect to computational unpredictability cannot be achieved in the standard model assuming indistinguishability obfuscation exists. Several 
refinements have been proposed since, including a statistical notion of unpredictability denoted by $\mathcal{S}^{\text {sup }}$ as well as source classes containing sources that are structurally required to produce output in a special way as well as sources which are restricted to only a fixed number of queries [BHK13b, BFM14, MH14b].

Our notion of UCE security strengthens the notion of unpredictability to what we call strong unpredictability and we denote the corresponding class of sources by $\mathcal{S}^{\text {s-cup }}$ for the computational variant and by $\mathcal{S}^{\text {s-sup }}$ for its statistical version. Namely, we demand that the leakage be computationally/statistically unpredictable even if the predictor additionally gets the answers to the queries that the source received from the oracle. We give the pseudo-code for strong unpredictability in Figure 3 .

It turns out that UCEs for strongly computationally unpredictable sources that can only make a single query (denoted by $\mathrm{UCE}\left[\mathcal{S}^{\text {s-cup }} \cap \mathcal{S}^{1 \text {-query }}\right]$ ) already imply hardcore functions for any one-way function. Furthermore, UCEs for strongly statistically unpredictable sources that can only make $q$ queries (denoted $\mathrm{UCE}\left[\mathcal{S}^{\text {s-sup }} \cap \mathcal{S}^{q \text {-query }}\right]$ ) imply $q$-query correlation-secure hash functions. We note that strongly unpredictable sources can be regarded as a generalization of so-called split sources BHK13b] which were introduced by BHK after the BFM impossibility results. We will discuss the exact relationship later.

So far UCEs have only been constructed in idealized models. BHK showed that a random oracle is UCE-secure in the strongest proposed settings and conjectured that HMAC is UCE-secure if the underlying compression function is modeled as an ideal function. This conjecture has recently been confirmed by Mittelbach [Mit14] who shows that HMAC and various Merkle-Damgård variants are UCEsecure in the ideal compression function model. We note that so far, no standard model instantiation of any (non-trivial) UCE variant has been proposed and, hence, we present the first standard model construction of UCEs 1 .

Techniques. Our construction is based on indistinguishability obfuscation and similar to many other recent constructions from iO [SW14, BST14, HSW14, BZ14, our construction also makes use of puncturable PRFs [SW14] which admit the generation of keys that allow to evaluate the PRF on all points except for points in a small target set (often containing just a single point). Our security reduction, however, differs from existing techniques. That is, we make use of point function obfuscations which allows us to hide the punctured points within our constructed circuits. Hiding the punctured points was also the key problem in the earlier discussed work by Bellare, Stepanovs and Tessaro BST14] who construct hardcore-functions for one-way functions. They solve the problem elegantly by using the one-way function from the security game to blind the punctured point by embedding the image under the one-way function. However, when testing whether a given point is equivalent to the punctured point this test is ambiguous which is why they need to assume differing-inputs obfuscators for one-way functions that map more than polynomially many points to the same image value. This is where point function obfuscation comes into the picture which allows us to bypass any assumptions related to differing-input obfuscation variants. Yet, of course, point obfuscators are as far as is currently known is an assumption incomparable to differing-inputs obfuscation.

POINT OBfuscation AND IO. In a recent and independent work, Hofheinz uses point obfuscation in a similar way to construct fully secure constrained pseudorandom functions [Hof14] in the random oracle model. A constrained PRF is a generalized form of a puncturable PRF which allows for the generation of keys that enable the holder to evaluate the PRF on a set of points but not on all points. In contrast to previous constructions [BW13, BGI14, KPTZ13] Hofheinz uses point obfuscation and an extension he introduces as extensible testers in conjunction with indistinguishability obfuscation to hide

\footnotetext{
${ }^{1}$ The UCE Framework is very flexible and it is, for example, possible to define a UCE restriction that corresponds to PRF security.
} 
which points a given key allows to honestly evaluate. This allows him to achieve full security without relying on complexity leveraging which was used in previous constructions entailing a superpolynomial loss of security in the adaptive setting. We note that unlike this work Hofheinz relies on the simpler assumption of plain point obfuscation (that is, obfuscation without auxiliary inputs) and he shows how to build extensible testers based on the DDH-based point obfuscator by Canetti [Can97.

Brzuska and Mittelbach study the connection between point obfuscation with multi-bit output secure in the presence of auxiliary inputs and indistinguishability obfuscation [BM14]. They show that indistinguishability obfuscation and a strong form of multi-bit point obfuscation are mutually exclusive. Their results do not carry over to the setting of statistically hard-to-invert auxiliary information (which we rely on for our construction of CIHs) and it is not clear if their results can be extended to cover plain AIPO, that is point functions with single-bit outputs.

OUR RESUlTs. We next discuss the specific UCE assumptions that our construction will meet and the relation to the specific point obfuscation schemes used. In Section 3 we will show that our construction is $\operatorname{UCE}\left[\mathcal{S}^{\text {s-cup }} \cap \mathcal{S}^{1 \text {-query }}\right]$-secure assuming iO, puncturable PRFs and the existence of AIPO. That is, we consider functions which are UCE-secure for computationally strongly unpredictable sources that make a single query. In Section 3.3, we prove that our construction is also $\operatorname{UCE}\left[\mathcal{S}^{\text {s-sup }} \cap \mathcal{S}^{q \text {-query }}\right]$-secure, that is, secure against statistically unpredictable sources that make at most $q$ queries.

As explained, we base the security of our construction on the existence of a different (incomparable) notion of point obfuscation. We consider a notion of AIPO which only needs to be secure against statistically unpredictable distributions but, in turn, we require it to be $q$-composable [CD08, BC10]. Intuitively, $q$-composability says that an obfuscation remains secure even if an adversary sees $q$ many (possibly related) obfuscations. The reason that we need $q$-composable AIPO is that now, the source is a allowed to make $q$ queries and hence, we need to hide $q$ points in the proof. $q$-composable AIPO implies multi-bit point function obfuscation [CD08] and thus does not exist, if iO exists [BM14].

However, as we here only consider sources in $\mathcal{S}^{\text {s-sup }}$, that is, sources which are only statistically strongly unpredictable, it suffices that our AIPO-notion is secure against statistically unpredictable samplers which weakens the notion of AIPO. Matsuda and Hanoka [MH14a] have recently shown that $q$-composable AIPO secure against statistically unpredictable samplers is implied by composable VGB-AI point obfuscators, a notion that Bitansky and Canetti constructed under the $q$-Strong Vector Decision Diffie Hellman assumption [BC10]. Note that, for the proof to work, we need to let the circuit size of our construction grow, artificially, with the number of queries $q$. Towards this goal, we use some padding that does not have any functionality.

In summary we get the following results:

\section{Theorem [informal].}

- Our construction is $\mathrm{UCE}\left[\mathcal{S}^{\text {s-cup }} \cap \mathcal{S}^{1 \text {-query }}\right]$-secure assuming indistinguishability obfuscation for all circuits in $\mathcal{P}$ /poly and AIPO secure with respect to computationally hard-to-invert auxiliary information exist.

- Our construction is $\mathrm{UCE}\left[\mathcal{S}^{\text {s-sup }} \cap \mathcal{S}^{q \text {-query }}\right]$-secure assuming indistinguishability obfuscation for all circuits in $\mathcal{P} /$ poly and q-composable AIPO with respect to statistically hard-to-invert auxiliary information exist.

On the FEASIBILITy OF OUR AIPO ASSUMPtions. Standard AIPO secure against computationally unpredictable samplers has been constructed by Canetti in [Can97] under (non-standard) variants of the DDH assumption and by Bitansky and Paneth in [BP12] under (non-standard) assumptions 
on pseudorandom permutations. We present the constructions and the underlying assumptions in Appendix A. One might hope that AIPO is naturally composable. However, Canetti et al. show that this is generally not the case [CD08, BC10]. On the other hand, Bitansky and Canetti [BC10] show that under the t-Strong Vector Decision Diffie Hellman assumption the original point obfuscation scheme of Canetti [Can97] composes in the so-called virtual grey-box (VGB) setting. The VGB setting was introduced by Bitansky and Canetti [BC10] and is a relaxation of the strongest obfuscation setting the virtual black-box (VBB) setting $\mathrm{BGI}^{+} 01, \mathrm{BGI}^{+} 12$. Similarly to $\mathrm{VBB}$ obfuscation, VGB obfuscation is in general not achievable, yet for the class of point functions it seems in reach [BC10]. The VGB setting is particularly interesting because "plain" VGB and VGB with auxiliary information are equivalent [BC10]. This result stands in contrast to the VBB setting where allowing auxiliary information results in a stronger notion. Furthermore, we currently have no candidate constructions for composable point obfuscation schemes in this stronger setting. We note that composable obfuscation in the VGB setting is sufficient for our purpose as Matsuda and Hanaoka MH14a show that this setting already implies $q$-composable AIPO with respect to statistically unpredictable samplers which form the basis for our q-query correlation-secure hash functions.

In a very recent work Brzuska and Mittelbach (BM) investigate the connection between indistinguishability obfuscation and multi-bit output point obfuscation secure in the presence of auxiliary input (MB-AIPO) [BM14]. A multi-bit point function $p_{x, m}$ is zero everywhere except on $x$ where it outputs $m$. BM show that various strong notions of MB-AIPO and indistinguishability obfuscation are mutually exclusive. However, their results do not seem to carry over to plain AIPO, that is to AIPO for plain point functions as needed in our constructions. We refer to [BM14 for a discussion on MB-AIPO and discuss the implications of an extension of the results of BM to plain AIPO shortly when talking about the feasibility of our UCE notions.

On the FEASIBILITy OF OUR UCE notions. In a recent work, Brzuska, Farshim, and Mittelbach (BFM; [BFM14]) show that, assuming indistinguishability obfuscation exists, no standard model hash construction can be UCE-secure with respect to computationally unpredictable sources. Our construction achieves a weaker yet related notion of security, namely UCE-security with respect to strongly computationally unpredictable sources which raises the question whether the BFM result can be extended to this setting.

The BFM result crucially hinges on the possibility of extending the output-length of the studied hash construction such that it is significantly larger than the key size. For example, this can be achieved by using multiple queries to the hash construction or via extending the output size by applying a pseudo-random generator [BFM14, BHK13c]. Both approaches fail with our construction: the size of our hash key grows with the number of allowed queries and since we consider strong unpredictability it seems implausible to prove the construction $\operatorname{PRG}(\mathrm{H}(\cdot, \cdot))$-secure under the assumption that $\mathrm{H}$ is UCE-secure with respect to strongly computationally unpredictable sources. Thus, we think that extending the BFM attack is implausible. Furthermore, if it can be extended this would immediately imply that indistinguishability obfuscation implies the non-existence of AIPO, which would be a surprising result. We discuss the BFM result in greater detail in Section 5 and note that, even if an extension of the BFM result were to break AIPOs with computational unpredictability, then the second construction would not be affected, as it only considers AIPOs secure with respect to statistically hard-to-invert auxiliary information.

Notions of Correlation-Secure Hash-Functions. We now compare our notion of q-query CIHs to different notions of correlated-input security. Note that q-query CIH means that the size of the hash-key can depend on the number of inputs $q$. However, and that is a crucial difference to 
previous works, each input value is hashed using the same hash-key. In turn, Freeman et al. FGK $^{+} 13$. as well as Rosen and Segev RS10] use a fresh hash-key for every input. Notably, the correlation-secure functions that they construct also have a trapdoor. Note that the correlated-input variant $2^{2}$ of the IND security game for deterministic public-key encryption [BFOR08, BBO07, BFO08] and the CIH game are almost identical if it is required that the CIH has a trapdoor. We can then view the computation of the $\mathrm{CIH}$ as an encryption operation and the $\mathrm{CIH}$ game becomes a slightly stronger version of the IND security game (that is, a real-or-random rather than a left-or-right game). Hence, a CIH function which has a trapdoor is also a deterministic public-key encryption scheme.

As in the schemes of $\left[\mathrm{FGK}^{+} 13, \mathrm{RS} 10\right]$ a new key needs to be generated for every new message, the constructions are not a deterministic public-key encryption scheme. In turn, if our q-query CIH were a trapdoor function, then by definition, it would also be a q-query deterministic public-key encryption scheme. Unfortunately, our construction of a q-query CIH does not come with a trapdoor, and we do not know whether this is possible.

Another related notion of CIH are statistically secure q-query CIHs. Here, as for our notion of q-query $\mathrm{CIH}$, the key size may grow with the number of queries and one uses the same hash key for each query. In contrast to our security notion one here requires that the output is statistically close to random given the hash key. As we are concerned with statistical security, this notion is only achievable for distributions that come with a notable amount of entropy, that is, the $q$ pre-images need to have entropy that is at least $q$ times the output length. In turn, for the notion of entropy that we consider, the entropy of the pre-images does not need to grow with $q$ and can also be less than the length of the output.

Hence, this notion of statistically secure CIH only applies to a substantially smaller class of distributions. In turn, while our construction relies on the strong assumption of indistinguishability obfuscation, statistically secure CIH can be achieved without any assumptions. That is, if the preimages carry enough (true) entropy, then one can extract $q$ uniformly random image values by using a $q$-wise independent hash-functions [FOR12].

Finally, Goyal, O'Neill, and Rao [GOR11] construct CIHs that are secure under polynomially related inputs and introduce a hierarchy of CIH notions: One-wayness under correlated inputs, unpredictability under correlated inputs and pseudorandomness under correlated inputs. These notions describe a hierarchy of security notions when we consider CIHs with superlogarithmic output length. We note that we achieve the strongest of these notions, namely pseudorandomness under correlated inputs.

\section{Preliminaries}

Notation. By $\lambda \in \mathbb{N}$, we denote the security parameter that we give to all algorithms implicitly in unary representation $1^{\lambda}$. By $\{0,1\}^{\ell}$ we denote the set of all bit-strings of length $\ell$, and by $\{0,1\}^{*}$ the set of all bit-strings of finite length. If $x, y \in\{0,1\}^{*}$ are two bit strings of the same length, then we denote their inner product over $\mathbb{G F}(2)$ by $\langle x, y\rangle$. The length of $x$ is denoted by $|x|$. For a finite set $X$, we denote the action of sampling $x$ uniformly at random from $X$ by $x \leftarrow \$ X$, and denote the cardinality of $X$ by $|X|$. We denote by $[i]$ the set $\{1, \ldots, i\}$. Algorithms are assumed to be randomized, unless otherwise stated. We call an algorithm efficient or PPT if it runs in time polynomial in the security parameter. If $\mathcal{A}$ is randomized then by $y \leftarrow \mathcal{A}(x ; r)$ we denote that $\mathcal{A}$ is run on input $x$ and with random coins $r$ and produced output $y$. If no randomness is specified, then we assume that $\mathcal{A}$ is run with freshly sampled uniform random coins, and write this as $y \leftarrow \$ \mathcal{A}(x)$. We often refer to algorithms, or tuples of algorithms, as adversaries. If $E$ is an event then we denote by $\operatorname{Pr}[E]$ its probability and

\footnotetext{
${ }^{2}$ Here, we refer to the variant where each message needs to have high entropy on its own, but might have low entropy conditioned on the other messages.
} 
if $X$ is a random variable, we denote its expectation by $\mathbb{E}[X]$. We write $X \mid E$ to denote the random variable $X$ conditioned on event $E$. We say a function $\operatorname{negl}(\lambda)$ is negligible if $\operatorname{negl}(\lambda) \in \lambda^{-\omega(1)}$. We say a function poly is polynomial if poly $\in \lambda^{\mathcal{O}(1)}$.

If we speak of an ensemble or a family $\left\{\mathcal{C}_{\lambda}\right\}_{\lambda \in \mathbb{N}}$ of circuits, denoted by a calligraphic letter such as $\mathcal{C}$, we mean that $\mathcal{C}_{\lambda}$ contains a set of circuits for each security parameter $\lambda \in \mathbb{N}$. We speak of a sequence of circuits $\left\{C_{\lambda}\right\}_{\lambda \in \mathbb{N}}$ to denote a non-uniform circuit, that is, one circuit for every security parameter. By a distribution or an ensemble of distributions $\mathcal{D}=\left\{D_{\lambda}\right\}_{\lambda \in \mathbb{N}}$ we identify a function ensemble $\left\{f_{\lambda}: S_{\lambda} \rightarrow[0,1]\right\}_{\lambda \in \mathbb{N}}$ with corresponding set $S_{\lambda}$ that assigns each element in $S_{\lambda}$ a probability weight in $[0,1]$ such that $\sum_{x \in S_{\lambda}} f_{\lambda}(x)=1$ for all $\lambda \in \mathbb{N}$. We consider only efficiently sampleable distributions $\mathcal{D}$ by which we mean that a (possibly non-uniform) algorithm Sam $_{\lambda}$ exists that on input a uniformly random string $r$ outputs a value in $S_{\lambda}$ according to distribution $D_{\lambda}$, that is, such that for all $\lambda \in \mathbb{N}$ and $x \in S_{\lambda}$

$$
\operatorname{Pr}_{r}\left[\operatorname{Sam}_{\lambda}(r)=x\right]=f_{\lambda}(x) .
$$

We often say we "run" a distribution or we simply write $D_{\lambda}\left(1^{\lambda}\right)$ to denote that the corresponding sample algorithm is invoked on fresh random coins.

\subsection{Obfuscation}

Obfuscation has a long tradition within cryptographic research and comes in many flavors. In the following section we present the various definitions that we use in this paper. We discuss constructions and candidates in Appendix A.

We start by recalling the strongest definition of virtual black-box (VBB) obfuscation with auxiliary inputs due to $\left.\mathrm{BGI}^{+} 01, \mathrm{GK} 05, \mathrm{BGI}^{+} 12\right]$.

Definition 2.1 (Worst-case obfuscator with auxiliary input (VBB-AI)). A PPT $\mathcal{O}$ is a worst-case obfuscator with auxiliary input for an ensemble $\mathcal{C}=\left\{\mathcal{C}_{\lambda}\right\}_{\lambda \in \mathbb{N}}$ of poly-size circuits if it satisfies:

- Functionality. For any $\lambda \in \mathbb{N}$ and $C \in \mathcal{C}_{\lambda}, \mathcal{O}(C)$ is a circuit which computes the same function as $C$, that is, for all $x$ it holds

$$
\operatorname{Pr}\left[C^{\prime}(x)=C(x) \mid C^{\prime} \leftarrow \$ \mathcal{O}(C)\right]=1 .
$$

- Polynomial slowdown. For any $\lambda \in \mathbb{N}$ and $C \in \mathcal{C}_{\lambda}, \operatorname{Pr}\left[\left|C^{\prime}\right| \leq \operatorname{poly}(|C|) \mid C^{\prime} \leftarrow{ }_{\$} \mathcal{O}(C)\right]=1$.

- Virtual black-box. For any PPT adversary $\mathcal{A}$ there is a PPT simulator Sim such that for all sufficiently large $\lambda \in \mathbb{N}, C \in \mathcal{C}_{\lambda}$ and $z \in\{0,1\}^{\text {poly }(\lambda)}$ :

$$
\left|\operatorname{Pr}[\mathcal{A}(z, \mathcal{O}(C))=1]-\operatorname{Pr}\left[\operatorname{Sim}^{C}\left(z, 1^{|C|}\right)=1\right]\right| \leq \operatorname{negl}(\lambda)
$$

where the probability is taken over the coins of $\mathcal{A}, \operatorname{Sim}$ and $\mathcal{O}$.

VBB obfuscation with auxiliary input requires that for any PPT adversary given the code of some functionality (and some auxiliary input) there exists a PPT simulator that given only black-box access to the functionality (and as input the same auxiliary input) produces a computationally indistinguishable distribution.

A provably weaker notion of obfuscation called virtual grey-box (VGB) was introduced by Bitansky and Canetti [BC10]. VGB is defined analogously to VBB with the exception that the simulator is given unbounded computation time but still restricted to only make polynomially many oracle queries. We will return to VGB obfuscation when discussing composition of so-called point function obfuscators. 
InDistinguishabiLity obfUSCATION. While VBB and VGB obfuscation as defined above provably do not exist in general for all circuits $\left[\mathrm{BGI}^{+} 01, \mathrm{BC} 10\right]$, weaker notions such as indistinguishability obfuscation may well exist. VBB requires the existence of a simulator. On the other hand, an indistinguishability obfuscation (iO) scheme only ensures that the obfuscations of any two functionally equivalent circuits are computationally indistinguishable. Indistinguishability obfuscation was originally proposed by Barak et al. [BGI ${ }^{+}$] as a potential weakening of virtual-black-box obfuscation. We recall the definition from $\mathrm{GGH}^{+} 13$.

Definition 2.2. A PPT algorithm $\mathrm{iO}$ is called an indistinguishability obfuscator for a circuit ensemble $\mathcal{C}=\left\{\mathcal{C}_{\lambda}\right\}_{\lambda \in \mathbb{N}}$ if the following conditions are satisfied:

- Correctness. For all security parameters $\lambda \in \mathbb{N}$, for all $C \in \mathcal{C}_{\lambda}$, and for all inputs $x$ we have that

$$
\operatorname{Pr}\left[C^{\prime}(x)=C(x): C^{\prime} \leftarrow \$ \mathrm{iO}\left(1^{\lambda}, C\right)\right]=1 .
$$

- Security. For any PPT distinguisher $\mathcal{D}$, for all pairs of circuits $C_{0}, C_{1} \in \mathcal{C}_{\lambda}$ such that $C_{0}(x)=$ $C_{1}(x)$ on all inputs $x$ the following distinguishing advantage is negligible:

$$
\left|\operatorname{Pr}\left[\mathcal{D}\left(1^{\lambda}, \mathrm{iO}\left(1^{\lambda}, C_{1}\right)\right)=1\right]-\operatorname{Pr}\left[\mathcal{D}\left(1^{\lambda}, \mathrm{iO}\left(1^{\lambda}, C_{0}\right)\right)=1\right]\right| \leq \operatorname{negl}(\lambda) .
$$

DifFERING-INPUTS OBFUSCATION. The notion of differing-inputs obfuscation is closely related to indistinguishability obfuscation and also goes back to the seminal paper of Barak et al. [BGI $\left.{ }^{+} 01\right]$. While indistinguishability obfuscation requires circuits to be identical on all inputs, differing-inputs obfuscation intuitively says that if a distinguisher can tell apart two obfuscated circuits then one can efficiently extract a value on which the circuits differ. We follow the definition of Ananth et al. $\mathrm{ABG}^{+} 13$ and Boyle et al. BCP14] and first define the notion of differing-inputs circuits.

Definition 2.3 (Differing-inputs circuits). A sample algorithm $\left(C_{0}, C_{1}, z\right) \leftarrow \$ \operatorname{Sam}\left(1^{\lambda}\right)$ that samples circuits from a circuit ensemble $\mathcal{C}=\left\{\mathcal{C}_{\lambda}\right\}_{\lambda \in \mathbb{N}}$ is said to be a differing-inputs distribution if for all PPT algorithms $\mathcal{A}$ there is a negligible function negl such that:

$$
\operatorname{Pr}\left[C_{0}(x) \neq C_{1}(x):\left(C_{0}, C_{1}, z\right) \leftarrow \$ \operatorname{Sam}\left(1^{\lambda}\right), x \leftarrow \$ \mathcal{A}\left(1^{\lambda}, C_{0}, C_{1}, z\right)\right] \leq \operatorname{negl}(\lambda)
$$

Definition 2.4 (Differing-inputs obfuscation). A PPT algorithm diO is a differing-inputs obfuscator for a differing-inputs distribution Sam (for circuit ensemble $\left\{\mathcal{C}_{\lambda}\right\}_{\lambda \in \mathbb{N}}$ ) if the following holds:

- Correctness. For all security parameters $\lambda \in \mathbb{N}$, for all $C \in \mathcal{C}_{\lambda}$, and for all inputs $x$ we have that

$$
\operatorname{Pr}\left[C^{\prime}(x)=C(x): C^{\prime} \leftarrow{ }^{\$} \operatorname{diO}\left(1^{\lambda}, C\right)\right]=1 .
$$

- Security. For any PPT distinguisher $\mathcal{D}$, for any $\left(C_{0}, C_{1}, z\right) \leftarrow \$ \operatorname{Sam}\left(1^{\lambda}\right)$ the following distinguishing advantage is negligible:

$$
\left|\operatorname{Pr}\left[\mathcal{D}\left(1^{\lambda}, \operatorname{diO}\left(1^{\lambda}, C_{1}\right), z\right)=1\right]-\operatorname{Pr}\left[\mathcal{D}\left(1^{\lambda}, \operatorname{diO}\left(1^{\lambda}, C_{0}\right), z\right)=1\right]\right| \leq \operatorname{negl}(\lambda) .
$$

The notion of differing-inputs obfuscation recently also gained much attention $\mathrm{ABG}^{+} 13$, $\mathrm{BCP} 14$, BP13. In particular, we will build on the work by Boyle, Chung and Pass [BCP14] who show that any general indistinguishability obfuscator also yields a mild version of a differing-inputs obfuscator. That is, any indistinguishability obfuscator for all circuits in $\mathcal{P} /$ poly is a also a differing-inputs obfuscator for circuits that differ on at most polynomially many inputs. We will use their result in a crucial way on circuits that differ on a single input. 
Theorem 2.5 ([BCP14 $]$ ). Let $\mathrm{iO}$ be an indistinguishability obfuscator for $\mathcal{P} /$ poly. Let $\left(\left\{\mathcal{C}_{\lambda}\right\}\right.$, Sam $)$ be a differing-inputs family for which there exists a polynomial $d: \mathbb{N} \rightarrow \mathbb{N}$, such that

$$
\operatorname{Pr}\left[\left|\left\{x: C_{0}(x) \neq C_{1}(x)\right\}\right| \leq d(\lambda) \mid\left(C_{0}, C_{1}, z\right) \leftarrow \$ \operatorname{Sam}\left(1^{\lambda}\right)\right] \geq 1-\operatorname{neg}(\lambda) .
$$

Then $\mathrm{iO}$ is a differing-inputs obfuscator for $\left(\left\{\mathcal{C}_{\lambda}\right\}\right.$, Sam).

Point obfuscation. While indistinguishability, as well as differing-inputs, obfuscation are obfuscation schemes for general circuits one can also study obfuscation schemes for particular function classes such as point functions. A point function $p_{x}$ for some value $x \in\{0,1\}^{*}$ is defined as

$$
p_{x}(s):= \begin{cases}1 & \text { if } s=x \\ \perp & \mathrm{o} / \mathrm{w}\end{cases}
$$

We consider a variant of point function obfuscators under auxiliary input which was first formalized by Canetti [Can97, although in a slightly different context. We here give the definition from [BP12] presented in a game based formulation. The first definition formalizes unpredictable distributions which are in turn used to define obfuscators for point functions.

Definition 2.6 (Unpredictable distribution). A distribution ensemble $\mathcal{D}=\left\{D_{\lambda}=\left(Z_{\lambda}, X_{\lambda}\right)\right\}_{\lambda \in \mathbb{N}}$, on pairs of strings is unpredictable if no poly-size (non-uniform) circuit can predict $X_{\lambda}$ from $Z_{\lambda}$. That is, for every poly-size circuit sequence $\left\{C_{\lambda}\right\}_{\lambda \in \mathbb{N}}$ and for all large enough $\lambda$ :

$$
\operatorname{Pr}_{(z, x) \leftrightarrow D_{\lambda}}\left[C_{\lambda}(z)=x\right] \leq \operatorname{negl}(\lambda)
$$

Remark. Alternatively, we could use a variant of Definition 2.6 for uniform distributions $\mathcal{D}$. Jumping ahead, we note that our positive result, Theorem 3.3 only requires AIPOs secure against uniform adversaries. For ease of presentation, we omit the explicit treatment of uniform and non-uniform adversaries.

Definition 2.7 (Auxiliary input point obfuscation for unpredictable distributions (AIPO)). A PPT algorithm AIPO is a point obfuscator for unpredictable distributions if if on input $(z, x)$ it outputs a polynomial-size circuit that returns 1 on $x$ and 0 everywhere else and satisfies the following secrecy property: for any (efficiently sampleable) unpredictable distribution $\mathcal{B}_{1}$ over $\{0,1\}^{\text {poly }(\lambda)} \times\{0,1\}^{\lambda}$ it holds for any PPT algorithm $\mathcal{B}_{2}$ that the probability that the following experiment outputs true for $\left(\mathcal{B}_{1}, \mathcal{B}_{2}\right)$ is negligibly close to $\frac{1}{2}$ :

$$
\begin{aligned}
& b \leftarrow \$\{0,1\} \\
& \left(z, x_{0}\right) \leftarrow \mathcal{B}_{1}\left(1^{\lambda}\right) \\
& x_{1} \leftarrow \$\{0,1\}^{\lambda} \\
& p \leftarrow \$ \operatorname{AIPO}\left(x_{b}\right) \\
& b^{\prime} \leftarrow \$ \mathcal{B}_{2}\left(1^{\lambda}, p, z\right) \\
& \text { return } b=b^{\prime}
\end{aligned}
$$

The probability is over the coins of adversary $\left(\mathcal{B}_{1}, \mathcal{B}_{2}\right)$, the coins of AIPO and the choices of $x_{1}$ and $b$. 
Composable VGB point obfuscation. The definition of AIPO requires that a single point obfuscation is secure. A natural question to ask is whether the scheme remains secure even if the adversary is allowed to see multiple obfuscations, possibly of related points. This leads to the study of composition of obfuscators and the version we consider in this work is composition by concatenation formalized by Lynn, Prabhakaran, and Sahai [LPS04]:

Definition 2.8 ( $t$-composable obfuscation [LPS04]). A PPT machine $\mathcal{O}$ is a $t$-composable obfuscator for a circuit ensemble $\mathcal{C}=\left\{\mathcal{C}_{\lambda}\right\}_{\lambda \in \mathbb{N}}$ if it satisfies the functionality and polynomial slow-down requirements, as in Definition 2.1, and for any PPT distinguisher $A$ and polynomial $p$, there is a simulator Sim, such that for any sequence of circuits $C^{1}, \ldots, C^{t} \in C_{\lambda}$ (where $t=\operatorname{poly}(\lambda)$ ), and any sufficiently large $\lambda$ :

$$
\left|\operatorname{Pr}\left[\mathcal{A}\left(\mathcal{O}\left(C^{1}\right), \ldots, \mathcal{O}\left(C^{t}\right)\right)=1\right]-\operatorname{Pr}\left[\operatorname{Sim}^{C^{1}, \ldots, C^{t}}\left({ }^{1}\left|C^{1}\right|, \ldots, 1{ }^{\mid C^{t}} \mid\right)=1\right]\right| \leq \frac{1}{p(\lambda)}
$$

where oracle $C^{1}, \ldots, C^{t}$ gets as input $(x, i)$ and returns $C^{i}(x)$.

Note that while [LPS04] consider t-composability in the VBB setting, we only require the relaxed VGB setting, that is, we allow the simulator to run in unbounded time. Interestingly, while VBB obfuscation in the presence of auxiliary input (AI) is a seemingly stronger requirement than plain VBB obfuscation, Bitansky and Canetti show that AI does not add any power to VGB. Note that in this setting we can only allow auxiliary input that statistically hides the target points, as the simulator could otherwise trivially recover the obfuscated points from the auxilliary input.

Proposition 2.9 ([BC10] $)$. Let $\mathcal{O}$ be a VGB obfuscator for a circuit ensemble $\mathcal{C}=\left\{\mathcal{C}_{\lambda}\right\}_{\lambda \in \mathbb{N}}$. Then $\mathcal{O}$ is also a VGB obfuscator with (statistically unpredictable) auxiliary input for the ensemble.

Bitansky and Canetti, furthermore, show that the point obfuscation scheme of Canetti [Can97] is a $t$ composable VGB point obfuscator under the $t$-Strong Vector Decision Diffie Hellman assumption [BC10]. Note that, as we can first compose and then introduce auxiliary input, this implies that under the t-Strong Vector Decision Diffie Hellman assumption Canetti's obfuscation scheme is also a VGB-AI point obfuscator. We recall the scheme by Canetti [Can97] in Appendix A.

From VGB BACK TO AIPO. In this work we develop techniques to work with AIPOs. In a recent work, Matsuda and Hanaoka [MH14a] relate the notions of VGB point obfuscators (resp. VGB-AI point obfuscators) and AIPO and show that composable VGB-AI point obfuscators imply the existence of composable AIPO with respect to statistically unpredictable distributions (Matsuda et al. call this notion AIND- $\delta$-sPUAI [MH14a]). Statistically unpredictable distributions are defined analogously to unpredictable distributions (Definition 2.6) with the exception that we allow the predictor to run in unbounded time.

\subsection{Universal Computational Extractors (UCE)}

The UCE Framework by Bellare, Hoang, and Keelveedhi (BHK; BHK13a]) introduces assumptions that allow us to instantiate random oracles in a wide range of applications and which are not succeptible to the impossibility result by Canetti, Goldreich and Halevi CGH98. Loosely speaking, UCEs are PRF-like assumptions that split the distinguisher into two parts: a first adversary $\mathrm{S}$ that gets access to a keyed hash function or a random oracle (and which is called the source), and a second adversary D that gets the hash key hk (and which is called the distinguisher). The two algorithms together try to guess whether the source was given access to a keyed hash function or to a random oracle.

Concretely, the UCE notions are defined via a two-stage UCE game (we depict the communication flow in Figure 1 and the pseudocode in Figure 2). First, the source $S$ is run with oracle access to HasH 


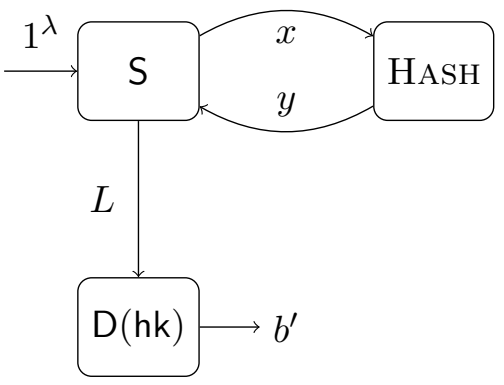

Figure 1: Schematic of the UCE game.

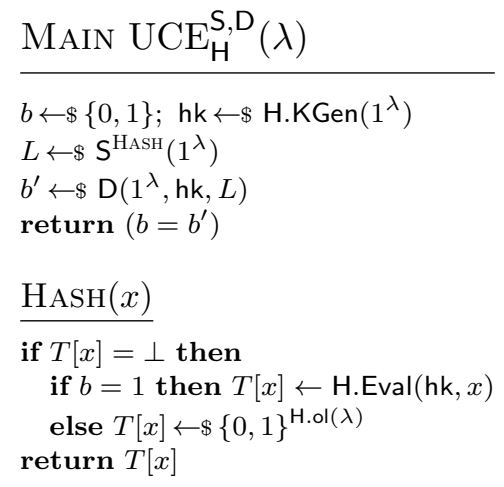

Figure 2: The UCE security game together with the unpredictability game (on the right). In the UCE game source $\mathrm{S}$ has access to HASH, which returns real or ideal hash values, and leaks $L$ to a distinguisher D. The latter additionally gets the hash key and outputs a bit $b^{\prime}$. On the right we give the unpredictability game.

to output some leakage $L$. Subsequently, distinguisher $\mathrm{D}$ is run on the leakage $L$ and hash key hk but without access to oracle HAsH. Distinguisher D outputs a single bit $b$ indicating whether oracle HASH implements a random oracle or hash function $\mathrm{H}$ with key hk.

Without any restrictions, (S, D) can easily win the UCE game. For example, say, source S makes a random query $x$ to receive $y \leftarrow \operatorname{HASH}(x)$ and outputs $(x, y)$ as leakage. As distinguisher $\mathrm{D}$ knows the hash key $\mathrm{hk}$ as well as the leakage $(x, y)$, it can recompute the hash value and check whether $y=\mathrm{H}(\mathrm{hk}, x)$. BHK present several possible restrictions on the source which give rise to various $\mathrm{UCE}$ notions.

Formal UCE Definition. In line with [BST14] we consider families of functions $F$ consisting of algorithms F.KGen, F.kl, F.Eval, F.il and F.ol. Algorithm F.KGen is a PPT algorithm taking the security parameter $1^{\lambda}$ and outputting a key $k \in\{0,1\}^{F . \mathrm{kl}(\lambda)}$ where $F . \mathrm{kl}: \mathbb{N} \rightarrow \mathbb{N}$ denotes the key length. Functions $F$.il $: \mathbb{N} \rightarrow \mathbb{N}$ and $F$.ol $: \mathbb{N} \rightarrow \mathbb{N}$ denote the input and output length functions associated to $F$ and for any $x \in\{0,1\}^{F . \mathrm{il}(\lambda)}$ and $k \leftarrow \$ F . \operatorname{KGen}\left(1^{\lambda}\right)$ we have that $F$.Eval $(k, x) \in\{0,1\}^{F . o l(\lambda)}$, where the PPT algorithm $F$.Eval denotes the "evaluation" function associated to $F$.

We denote hash functions by H. Let $\mathrm{H}=(\mathrm{H} . \mathrm{KGen}, \mathrm{H}$. Eval, H.kl, H.il, H.ol) be a hash-function family and let (S, D) be a pair of PPT algorithms. We define the UCE advantage of a pair (S, D) against $\mathrm{H}$ through

$$
\operatorname{Adv}_{\mathrm{H}, \mathrm{S}, \mathrm{D}}^{\text {uce }}(\lambda):=2 \cdot \operatorname{Pr}\left[\operatorname{UCE}_{\mathrm{H}}^{\mathrm{S}, \mathrm{D}}(\lambda)\right]-1,
$$

where game $\operatorname{UCE}_{H}^{\mathrm{S}, \mathrm{D}}(\lambda)$ is shown in Figure 2 on the left (in Figure 1 we give a schematic overview of the communication within the game).

UnPREDICTABILITY. Without any further restrictions there are PPT pairs (S, D) that achieve an advantage in the $\mathrm{UCE}_{\mathrm{H}}^{\mathrm{S}, \mathrm{D}}(\lambda)$ game close to 1 . BHK define several possible restrictions for sources yielding various flavors of UCE assumptions [BHK13a]. Here, we are interested in a strengthened version of the original computational unpredictability BHK13a] restriction. A source $\mathrm{S}$ is called computationally unpredictable if the advantage of any PPT predictor P, defined by

$$
\operatorname{Adv}_{S, P}^{\text {pred }}(\lambda):=\operatorname{Pr}\left[\operatorname{Pred}_{S}^{\mathrm{P}}(\lambda)\right],
$$


is negligible, where game $\operatorname{Pred}_{S}^{\mathrm{P}}(\lambda)$ is shown in Figure 2 on the right. In line with [BHK13b], we call the class of all computationally unpredictable sources $\mathcal{S}^{\text {cup }}$, where $\mathcal{S}^{\text {cup }}$ denotes the class (set) of all computationally unpredictable sources. Similarly, we define the class of statistically unpredictable sources where the predictor in $\operatorname{game~}_{\operatorname{Pred}}^{\mathrm{P}}(\lambda)$ can run in unbounded time but is still restricted to only polynomially many oracle queries. The class of statistically unpredictable sources is denoted by $\mathcal{S}^{\text {sup }}$.

UCE SECURITy. We say a hash function $\mathrm{H}$ is $\mathrm{UCE}$ secure for sources $\mathrm{S} \in \mathcal{S}$ denoted by $\mathrm{UCE}[\mathcal{S}]$, if for all PPT sources $S \in \mathcal{S}$ and all PPT distinguishers $\mathrm{D}$ the advantage $\operatorname{Adv}_{\mathrm{H}, \mathrm{S}, \mathrm{D}}^{\mathrm{uce}}(\lambda)$ is negligible. In that way we get the UCE assumptions $\mathrm{UCE}\left[\mathcal{S}^{\text {cup }}\right]$ and $\mathrm{UCE}\left[\mathcal{S}^{\text {sup }}\right]$, that is, UCE with respect to computationally (resp. statistically) unpredictable sources ${ }^{3}$

\subsection{Puncturable PRFs}

Besides point function obfuscation schemes, our main ingredient in the upcoming proofs are so-called puncturable pseudorandom functions (PRF) [SW14]. A family of puncturable PRFs $G:=(G$.KGen, G.Puncture, G.kl, G.Eval, G.il, G.ol) consists of functions that specify input length, output length and key length as well as a key generation algorithm $k \leftarrow G$.KGen, a deterministic evaluation algorithm $G$.Eval $(k, x)$ that takes a key $k$, an input $x$ of length $G$.il $\left(1^{\lambda}\right)$ and outputs a value $y$ of length $G$.ol $\left(1^{\lambda}\right)$. Additionally, there is a PPT puncturing algorithm $G$.Puncture which on input a polynomial-size set $S \subseteq\{0,1\}^{G . i l(\lambda)}$, outputs a special key $k_{S}$. A family of functions is called puncturable PRF if the following two properties are observed

- Functionality preserved under puncturing. For every PPT adversary $\mathcal{A}$ such that $\mathcal{A}\left(1^{\lambda}\right)$ outputs a polynomial-size set $S \subseteq\{0,1\}^{G \text {.il }(\lambda)}$, it holds for all $x \in\{0,1\}^{G \text {.il }(\lambda)}$ where $x \notin S$ that:

$$
\operatorname{Pr}\left[G . \operatorname{Eval}(k, x)=G \cdot \operatorname{Eval}\left(k_{S}, x\right): k \leftarrow \$ G \cdot \operatorname{KGen}\left(1^{\lambda}\right), k_{S} \leftarrow \$ G . P u n c t u r e(k, S)\right]=1
$$

- Pseudorandom at punctured points. For every PPT adversary $\left(\mathcal{A}_{1}, \mathcal{A}_{2}\right)$ such that $\mathcal{A}_{1}\left(1^{\lambda}\right)$ outputs a set $S \subseteq\{0,1\}^{G . i l(\lambda)}$ and state st, consider an experiment where $k \leftarrow G$.KGen $\left(1^{\lambda}\right)$ and $k_{S}=G$.Puncture $(k, S)$. Then we have

$$
\mid \operatorname{Pr}\left[\mathcal{A}_{2}\left(\text { st }, k_{S}, S, G . \operatorname{Eval}(k, S)\right)=1\right]-\operatorname{Pr}\left[\mathcal{A}_{2}\left(\text { st }, k_{S}, S, U_{G . \mathrm{ol}(\lambda) \cdot|S|}\right)=1\right] \mid \leq \operatorname{negl}(\lambda)
$$

where $\operatorname{Eval}(k, S)$ denotes the concatenation of $\operatorname{Eval}\left(k, x_{1}\right), \ldots, \operatorname{Eval}\left(k, x_{k}\right)$ where $S=\left\{x_{1}, \ldots, x_{k}\right\}$ is the enumeration of the elements of $S$ in lexicographic order, negl is a negligible function, and $U_{\ell}$ denotes the uniform distribution over $\{0,1\}^{\ell}$.

As observed by BW13, BGI14, KPTZ13] puncturable PRFs can, for example, be constructed from pseudorandom generators via the GGM tree-based construction [GGM84. Note that, as AIPO implies one-way functions (see Lemma B.1) AIPO, thus, also implies the existence of puncturable PRFs.

\section{UCEs from iO and Point Obfuscation}

In this section we present our constructions of UCEs from $\mathrm{iO}$ and AIPO. We first define the precise UCE notions that our constructions achieve and introduce the UCE restriction of strong unpredictability. We will then in Section 3.2 present a construction of a UCE-secure function with respect to sources which

\footnotetext{
${ }^{3}$ The notion $\mathrm{UCE}\left[\mathcal{S}^{\text {cup }}\right]$ was originally named $\mathrm{UCE} 1$ and later changed to $\mathrm{UCE}\left[\mathcal{S}^{\text {cup }}\right]$ BHK13a, BHK13b]. The notion of statistical unpredictability was introduced in BFM14, BHK13b.
} 


\begin{tabular}{l} 
MAIN stPred $\mathrm{S}(\lambda)$ \\
\hline$X^{*}, Y^{*} \leftarrow \emptyset$ \\
$L \leftarrow \$ \mathrm{~S}^{\mathrm{HASH}}\left(1^{\lambda}\right)$ \\
$x^{\prime} \leftarrow \$ \mathrm{P}^{\mathrm{HASH}}\left(1^{\lambda}, L, Y^{*}\right)$ \\
return $\left(x^{\prime} \in X^{*}\right)$ \\
$\operatorname{HASH}(x)$ \\
\hline$X^{*} \leftarrow X^{*} \cup\{x\}$ \\
$y \leftarrow \$\{0,1\}^{\mathrm{H} . \mathrm{ol}(\lambda)}$ \\
$Y^{*} \leftarrow Y^{*} \cup\{y\}$ \\
return $y$
\end{tabular}

Splt Source $\mathrm{S}^{\mathrm{HASH}}\left(1^{\lambda}\right)$
$\left(L_{0}, \mathbf{x}\right) \leftarrow \$ \mathrm{~S}_{0}\left(1^{\lambda}\right)$
for $i=1, \ldots,|\mathbf{x}|$ do $\mathbf{y}[i] \leftarrow \$ \operatorname{HASH}(\mathbf{x}[i])$
$L_{1} \leftarrow \$ \mathrm{~S}_{1}\left(1^{\lambda}, \mathbf{y}\right) ; L \leftarrow\left(L_{0}, L_{1}\right)$
return $L$

Figure 3: On the left: the strong unpredictability game where the predictor, in addition to the leakage is also given the result of the HASH queries. On the right: the definition of split sources BHK13b. A split source $\mathrm{S}=\mathrm{Splt}\left[S_{0}, S_{1}\right]$ consists of two parts $S_{0}$ and $S_{1}$ that jointly generate leakage $L$ and neither part gets direct oracle access to HASH. Note that the BFM attack BFM14] applies to split sources as presented above (see discussion in Section 3.1.

are strongly computationally-unpredictable and which make exactly one oracle query. In Section 3.3 we will show how to extend the construction to allow for an a-priory fixed number of queries by switching to a statistical version of strong unpredictability.

Interestingly, our construction for both cases is basically the same modulo circuit padding. That is, our constructions depend on an obfuscation of a circuit, which in both cases is the same but padded to a different length. A larger but functionally equivalent circuit seems to be necessary to allow for multiple source queries.

We will discuss applications of our constructions in Section 4 . In Section 5 we will discuss why our construction does not (seem to) fall pray to the BFM attacks on computationally unpredictable sources [BFM14].

\subsection{Strongly Unpredictable and $q$-Query Sources}

We now introduce the precise source restrictions for our upcoming UCE constructions. We define a new restriction that we call strong unpredictability and which can be seen as either a stronger form of unpredictability or a relaxed version of split sources. Secondly, we consider sources that make only a bounded number of oracle queries.

Strong UnPREDictability. We consider sources which are strongly unpredictable both in the computational and in the statistical sense. We denote by $\mathcal{S}^{\text {s-cup }}$ the class of sources which are strongly, computationally unpredictable and by $\mathcal{S}^{\text {s-sup }}$ the class of strongly, statistically unpredictable sources. Strong unpredictability is a stronger requirement than unpredictability and we require that the leakage hides queries to HASH even if the predictor is given the query results. We say that a source $\mathrm{S}$ is called strongly computationally unpredictable if the advantage of any PPT predictor P, defined by

$$
\operatorname{Adv}_{S, P}^{\text {stpred }}(\lambda):=\operatorname{Pr}\left[\operatorname{stPred}_{S}^{P}(\lambda)\right],
$$

is negligible, where game $\operatorname{stPred}_{\mathrm{S}}^{\mathrm{P}}(\lambda)$ is shown in Figure 3 on the left. For the case of strongly statistically unpredictable sources $\left(\mathcal{S}^{\text {s-sup }}\right)$ we allow the predictor to be unbounded in its running time, but restrict the number of oracle queries to be bounded polynomially.

In order to circumvent the BFM attacks on computationally unpredictable sources BHK introduce the notion of split sources BHK13b, BFM14]. A source $\mathrm{S}$ is called split source, denoted by $\mathrm{S} \in \mathcal{S}^{\text {splt }}$ 
if it can be decomposed into two algorithms $S_{0}$ and $S_{1}$ such that neither part gets direct access to oracle Hash. We give the pseudocode of split sources in Figure 3 on the right. In a first step algorithm $\mathrm{S}_{0}$ outputs a leakage string $L_{0}$ together with a vector $\mathbf{x}$. Then, each of the entries in $\mathbf{x}$ is queried to HASH and the results stored in vector $\mathbf{y}$. Finally, the second algorithm $\mathrm{S}_{1}$ is run on vector $\mathbf{y}$ to produce the second part of the leakage $L_{1}$.

There is one intricacy with split sources that we discovered when discussing strong unpredictability with Mihir Bellare Bel14. $S_{0}$ outputs a list $\mathbf{x}$ that is then used to generate the input to $S_{1}$ as

$$
\text { for } i=1, \ldots,|\mathbf{x}| \text { do } \mathbf{y}[i] \leftarrow \operatorname{HASH}(\mathbf{x}[i])
$$

As $\mathbf{x}$ is a list it allows for duplicate values. This, however, means that $\mathrm{S}_{0}$ can communicate arbitrary values to $S_{1}$ by encoding them using duplicates (e.g., using the two identical values to encode a 0 and two different values to encode a 1). The result is that the attack due to BFM also applies to split sources. A simple solution to recover split sources is to disallow duplicates in vector $\mathbf{x}$. This yields the following formulation which we henceforth associate with split sources:

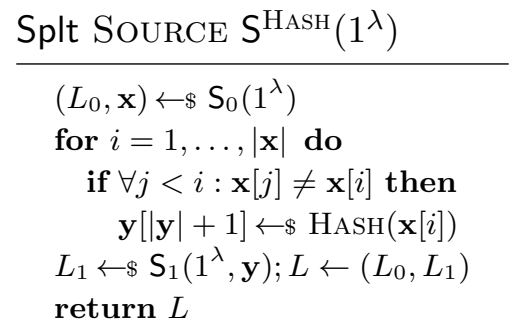

We note that when considering only single-query split sources, which suffice for the only known application (universal hardcore functions, see Section 4) then both formulations are equivalent.

One can prove that split sources are a (strict) subclass of strongly unpredictable sources, that is, $\mathcal{S}^{\text {splt }} \cap \mathcal{S}^{\text {cup }} \subseteq \mathcal{S}^{\text {s-cup }}$ (and similarly in the statistical case $\mathcal{S}^{\text {splt }} \cap \mathcal{S}^{\text {sup }} \subseteq \mathcal{S}^{\text {s-sup }}$ ). For this note that the leakage $L_{0}$ of the first algorithm of a split source is independent of any oracle answers. Similarly, if the oracle is implemented by a random oracle (which is the case in the unpredictability experiment) then the leakage $L_{1}$ of the second algorithm is independent of any actual oracle query. The inclusion is strict. Consider, for example, a source that queries HASH on $x$ to receive $y$ to then output $P R F_{x}(y)$ that is the image of a pseudorandom function at point $y$ under key $x$. For the case of statistical unpredictability consider the source that outputs $x \oplus y$. Both distributions cannot be simulated by a split source. This yields the following lemma:

Lemma 3.1. The class of split sources is a strict subclass of strongly unpredictable sources:

$$
\mathcal{S}^{\text {splt }} \cap \mathcal{S}^{\text {cup }} \subsetneq \mathcal{S}^{\text {s-cup }} \quad \text { and } \quad \mathcal{S}^{\text {splt }} \cap \mathcal{S}^{\text {sup }} \subsetneq \mathcal{S}^{\text {s-sup }}
$$

We formally prove Lemma 3.1 for the statistical case. The computational case follows analogously.

Proof. We have already seen that there are sources in $\mathcal{S}^{\text {s-sup }}$ that are not in $\mathcal{S}^{\text {splt }}$. It thus remains to show that any source in $\mathcal{S}^{\text {splt }} \cap \mathcal{S}^{\text {sup }}$ is also a strongly, statistically unpredictable source. We assume that we are always in the random oracle setting, that is, HASH is implemented by a random oracle. Note that this is without loss of generality since membership in $\mathcal{S}^{\text {s-sup }}$ is only defined via the strong unpredictability game which is in the random oracle setting.

Let $\mathrm{S}=\left(\mathrm{S}_{0}, \mathrm{~S}_{1}\right)$ be a source in $\mathcal{S}^{\text {splt }} \cap \mathcal{S}^{\text {sup }}$ and assume there exists a predictor $\mathrm{P}$ in the strong unpredictability game. We construct $\mathrm{P}^{\prime}$ in the plain unpredictability game. Predictor $\mathrm{P}^{\prime}$ gets as input leakage $L=\left(L_{0}, L_{1}\right)$. It guesses the number of queries $q$ that were made by source $\mathrm{S}_{0}$ and constructs a vector $\mathbf{y}^{\prime}$ consisting of $q$ random values of length $\mathrm{H}$.ol $(\lambda)$. It then runs source $\mathrm{S}_{1}$ on input $\mathbf{y}^{\prime}$ to receive leakage $L_{1}^{\prime}$. Finally, it runs predictor $\mathrm{P}$ on input $\left(\left(L_{0}, L_{1}^{\prime}\right), Y^{*}\right)$ where $Y^{*}$ is a random permutation of the values in $\mathbf{y}^{\prime}$. It outputs whatever $\mathrm{P}$ outputs. 
AnALYSis. As the unpredictability game (as well as the strong unpredictability game) is in the random oracle setting, the simulation of the input for $S_{1}$ is perfect in case $\mathrm{P}^{\prime}$ guesses the correct number of queries. In this case leakage $\left(L_{0}, L_{1}\right)$ and $\left(L_{0}, L_{1}^{\prime}\right)$ are distributed identically and the simulation for $\mathrm{P}$ is perfect and hence

$$
\operatorname{Adv}_{\mathrm{S}, \mathrm{P}^{\prime}}^{\text {pred }}(\lambda)=\frac{1}{\max _{q}} \cdot \mathcal{A}_{\mathrm{S}, \mathrm{P}}^{\text {stpred }}(\lambda)
$$

where $\max _{q}$ is an upper bound on the maximum number of queries of source $\mathrm{S}_{0}$.

q-QUERY UCE. Our first construction will only admit sources which make exactly one query. We call such sources single-query sources and denote the corresponding source class by $\mathcal{S}^{1 \text {-query }}$. We also consider a relaxed notion to allow for polynomially bounded number of queries for some polynomial $q:=q(\lambda)$. We call the corresponding sources $q$-query sources and denote their source class by $\mathcal{S}^{q-q u e r y}$. We note that sources restricted to a constant number of queries are discussed in [BHK13b].

\subsection{A UCE Construction Secure Against Sources in $\mathcal{S}^{\text {s-cup }} \cap \mathcal{S}^{1 \text {-query }}$}

We will now present our construction which depending on different assumptions on the existence of point obfuscators will achieve $\operatorname{UCE}\left[\mathcal{S}^{\text {s-cup }} \cap \mathcal{S}^{1 \text {-query }}\right]$-security or $\operatorname{UCE}\left[\mathcal{S}^{\text {s-sup }} \cap \mathcal{S}^{q \text {-query }}\right]$-security. Note that depending on the number of supported queries the construction needs to pad the circuit before obfuscating it.

Construction 3.2. Let $s: \mathbb{N} \rightarrow \mathbb{N}$, let $G$ be a puncturable PRF and let $\mathrm{iO}$ be an indistinguishability obfuscator for all circuits in $\mathcal{P} /$ poly. We define our hash function family $\mathrm{H}$ as

\begin{tabular}{|c|c|}
\hline H.KGen $\left(1^{\lambda}\right)$ & H.Eval(hk, $x)$ \\
\hline $\begin{array}{l}k \leftarrow \$ G \cdot \operatorname{KGen}\left(1^{\lambda}\right) \\
\mathrm{hk} \leftarrow \$ \mathrm{iO}(\operatorname{PAD}(s(\lambda), G \cdot \operatorname{Eval}(k, \cdot)))\end{array}$ & $\begin{array}{l}\bar{C} \leftarrow \mathrm{hk} \\
\text { return } \bar{C}(x)\end{array}$ \\
\hline
\end{tabular}

where PAD : $\mathbb{N} \times\{0,1\}^{*} \longrightarrow\{0,1\}^{*}$ denotes a deterministic padding algorithm that takes as input an integer and a circuit and outputs a functionally equivalent circuit padded to length $s(\lambda) 4^{4}$

In other words, the key generation algorithm H.KGen $\left(1^{\lambda}\right)$ runs $k \leftarrow G$.KGen $\left(1^{\lambda}\right)$ and returns $\mathrm{iO}(G$.Eval $(k, \cdot))$, i.e., an obfuscation of the evaluation circuit of PRF $G$ with key $k$ hardwired into it. Function H.Eval is basically a universal Turing machine which runs input $x$ on the obfuscated circuit hk.

Theorem 3.3. If $G$ is a secure puncturable PRF, if $\mathrm{iO}$ is a secure indistinguishability obfuscator and if AIPO exists, then the hash function family $\mathrm{H}$ defined in Construction 3.2 is $\mathrm{UCE}\left[\mathcal{S}^{\text {s-cup }} \cap \mathcal{S}^{1 \text {-query }}\right]$-secure.

We prove the theorem via a sequence of 5 games (depicted in Figure 4) where game Game denotes the original $\mathrm{UCE}\left[\mathcal{S}^{\text {s-cup }} \cap \mathcal{S}^{1 \text {-query }}\right]$ game with hidden bit $b$ fixed to 1 . We first present the games and subsequently the analysis of the individual game hops. Let $\mathrm{S} \in \mathcal{S}^{\text {s-cup }} \cap \mathcal{S}^{1 \text {-query }}$.

Game $_{1}$ : The first game is the original $\operatorname{UCE}\left[\mathcal{S}^{\text {s-cup }} \cap \mathcal{S}^{1 \text {-query }}\right]$-game. Here, the hash key hk is an obfuscation of the circuit $C_{1}[k](x):=G$. Eval $(k, x)$ (see Figure 4 ) where $k$ is a key for the puncturable PRF.

\footnotetext{
${ }^{4}$ Function $s$ needs to be chosen in accordance with the puncturable PRF to allow for the required number of puncturings.
} 


\begin{tabular}{|c|c|c|c|c|c|}
\hline $\operatorname{Game}_{1}(\lambda)$ & $\operatorname{Game}_{2}(\lambda)$ & \multicolumn{2}{|r|}{$\mathrm{iO}$} & \multicolumn{2}{|c|}{$\mathrm{AIPO}+\mathrm{iO}+[\mathrm{BCP} 14$} \\
\hline$x^{*}, y^{*} \leftarrow \perp$ & $x^{*}, y^{*} \leftarrow \perp$ & $x^{*}, y^{*} \leftarrow$ & & $x^{*}, y^{*} \leftarrow \perp$ & $x^{*}, y^{*} \leftarrow \perp$ \\
\hline$k \leftarrow \$ G . K \operatorname{Ken}\left(1^{\lambda}\right)$ & $k \leftarrow \$ G . K \operatorname{Ken}\left(1^{\lambda}\right)$ & $k \leftarrow \$ G . K C$ & $\operatorname{Sen}\left(1^{\lambda}\right)$ & $k \leftarrow \$ G . \operatorname{KGen}\left(1^{\lambda}\right)$ & $k \leftarrow \$ G . \mathrm{KGen}\left(1^{\lambda}\right)$ \\
\hline \multirow{3}{*}{$L \leftarrow \$ \mathrm{~S}^{\mathrm{HASH}}\left(1^{\lambda}\right)$} & $L \leftarrow \$ \mathrm{~S}^{\mathrm{HASH}}\left(1^{\lambda}\right)$ & $L \leftarrow \$ \mathrm{~S}^{\mathrm{HAS}}$ & ${ }^{\mathrm{H}}\left(1^{\lambda}\right)$ & $L \leftarrow \$ \mathrm{~S}^{\mathrm{HASH}}\left(1^{\lambda}\right)$ & $L \leftarrow \$ \mathrm{~S}^{\mathrm{HASH}}\left(1^{\lambda}\right)$ \\
\hline & $p \leftarrow \$ \operatorname{AIPO}\left(x^{*}\right)$ & $p \leftarrow \$$ AIPC & $\left(x^{*}\right)$ & $p \leftarrow \$ \operatorname{AIPO}\left(x^{*}\right)$ & \\
\hline & $k^{*} \leftarrow G$.Puncture $\left(k, x^{*}\right)$ & $k^{*} \leftarrow G . P$ & uncture $\left(k, x^{*}\right)$ & & \\
\hline $\mathrm{hk} \leftarrow \$ \mathrm{iO}\left(C_{1}[k]\right)$ & $\mathrm{hk} \leftarrow \$ \mathrm{iO}\left(C_{2}\left[k^{*}, p, y^{*}\right]\right)$ & $\mathrm{hk} \leftarrow \$ \mathrm{iO}($ & $\left.C_{2}\left[k^{*}, p, y^{*}\right]\right)$ & $\mathrm{hk} \leftarrow \$ \mathrm{iO}\left(C_{3}\left[k, p, y^{*}\right]\right)$ & $\mathrm{hk} \leftarrow \$ \mathrm{iO}\left(C_{4}[k]\right)$ \\
\hline$b^{\prime} \leftarrow \$ \mathrm{D}\left(1^{\lambda}, \mathrm{hk}, L\right)$ & $b^{\prime} \leftarrow \$ \mathrm{D}\left(1^{\lambda}, \mathrm{hk}, L\right)$ & $b^{\prime} \leftarrow \$ \mathrm{D}(1$ & $, \mathrm{hk}, L)$ & $b^{\prime} \leftarrow \$ \mathrm{D}\left(1^{\lambda}, \mathrm{hk}, L\right)$ & $b^{\prime} \leftarrow \$ \mathrm{D}\left(1^{\lambda}, \mathrm{hk}, L\right)$ \\
\hline return $\left(1=b^{\prime}\right)$ & return $\left(1=b^{\prime}\right)$ & return $(1$ & $\left.=b^{\prime}\right)$ & return $\left(1=b^{\prime}\right)$ & return $\left(1=b^{\prime}\right)$ \\
\hline $\operatorname{HASH}(x)$ & $\operatorname{HASH}(x)$ & $\operatorname{HASH}(x)$ & & $\operatorname{HASH}(x)$ & $\operatorname{HASH}(x)$ \\
\hline$x^{*} \leftarrow x$ & $x^{*} \leftarrow x$ & $x^{*} \leftarrow x$ & & $x^{*} \leftarrow x$ & $x^{*} \leftarrow x$ \\
\hline$y^{*} \leftarrow G \cdot \operatorname{Eval}(k, x)$ & $y^{*} \leftarrow G \cdot \operatorname{Eval}(k, x)$ & $y^{*} \leftarrow \$\{0$ & $1\}^{\mathrm{H} . \mathrm{ol}(\lambda)}$ & $y^{*} \leftarrow \$\{0,1\}^{\mathrm{H} . \mathrm{ol}(\lambda)}$ & $y^{*} \leftarrow \$\{0,1\}^{\mathrm{H} . \mathrm{ol}(\lambda)}$ \\
\hline return $y^{*}$ & return $y^{*}$ & return & & return $y^{*}$ & return $y^{*}$ \\
\hline Circuit $C_{1}[k](x)$ & \multicolumn{2}{|c|}{ Circuit $C_{2}\left[k^{*}, p, y^{*}\right](x)$} & \multicolumn{2}{|c|}{ Circuit $C_{3}\left[k, p, y^{*}\right](x)$} & 2CUIT $C_{4}[k](x)$ \\
\hline return $G . \operatorname{Eval}(k, x)$ & \multicolumn{2}{|c|}{$\begin{array}{l}\text { if } p(x)=\perp \text { then } / / \text { if } x \neq x^{*} \\
\text { return } G . \operatorname{Eval}\left(k^{*}, x\right) \\
\text { return } y^{*}\end{array}$} & \multicolumn{2}{|c|}{$\begin{array}{l}\text { if } p(x)=\perp \text { then } \\
\text { return } G . \operatorname{Eval}(k, x) \\
\text { return } y^{*}\end{array}$} & ceturn $G \cdot \operatorname{Eval}(k, x)$ \\
\hline
\end{tabular}

Figure 4: The games used in the proof of Theorem 3.3 on the top and the used circuits on the bottom. To highlight the changes from game to game we have marked the changed lines with a light gray background color. By $C[k](x)$ we denote that circuit $C$ depends on $k$ (during construction time) and takes $x$ as input. The arrows above the games indicate the security reduction to get from Game $i$ to Game $_{i+1}$.

$\mathrm{Game}_{2}$ : Let $x^{*}$ be the single query that the source $\mathrm{S}$ makes to its HASH oracle and let $y^{*}:=$ $G$. Eval $\left(k, x^{*}\right) . G$ Game $_{2}$ is similar to Game 1 except that we puncture the PRF at $x^{*}$. Namely, the hash key hk does not consist of an obfuscation of $C_{1}[k]$ anymore, but rather of an obfuscation of the circuit $C_{2}\left[k^{*}, p, y^{*}\right]$. The circuits $C_{1}[k]$ and $C_{2}\left[k^{*}, p, y^{*}\right]$ are functionally equivalent. However, instead of the normal PRF key, $C_{2}$ uses a punctured PRF key $k^{*}$ which is punctured at value $x^{*}$ (or equivalently, at all values $x$ where $p(x)=1$ ). Here, $p$ is computed as the AIPO obfuscation of the point function $p_{x^{*}}$ and hence, $p(x)=1$ if and only if $x$ is equal to the single hash query $x^{*}$ of the source. On input a value $x$, circuit $C_{2}\left[k^{*}, p, y^{*}\right]$ checks whether $p(x)=\perp$ (i.e., if $\left.x \neq x^{*}\right)$ : if so, it returns $G$.Eval $\left(k^{*}, x\right)$, otherwise it outputs $y^{*}$.

Game 3 : The game is equivalent to Game 2 except that oracle HasH now samples $y^{*}$ uniformly at random instead of invoking $G$. Eval $(k,$.$) . Note that C_{2}\left[k^{*}, p, y^{*}\right]$ is parametrized by $y^{*}$.

Game $_{4}$ : The game is equivalent to the previous game except that now an obfuscation of circuit $C_{3}\left[k, p, y^{*}\right]$ is used as hash key hk. Circuit $C_{3}\left[k, p, y^{*}\right]$ is identical to circuit $C_{2}\left[k^{*}, p, y^{*}\right]$, except that it uses the original PRF key $k$ instead of the punctured key $k^{*}$. Note that circuits $C_{3}\left[k, p, y^{*}\right]$ and $C_{2}\left[k^{*}, p, y^{*}\right]$ have identical input-output behaviour.

Game 5 : The game is equivalent to the previous game except that now an obfuscation of circuit $C_{4}[k]$ is used as hash key hk. Circuit $C_{4}[k]$ is our original circuit again, that is, $C_{4}[k](\cdot):=G$. Eval $(k, \cdot)$. Game $_{5}$ is our intended target. It is the UCE-security game for our construction in the random oracle world (that is, oracle HASH implements a random oracle).

In Game $_{5}$ we are in an identical setting to the UCE-game with the hidden bit set to 0 . That is, the HASH oracle answers with randomly chosen values independent of the hash key. Further note, that $C_{4}$ 
and $C_{1}$ are identical, that is they are as in the construction. Thus, we can write the advantage of an adversary (S, D) in the UCE-security game as

$$
\begin{aligned}
\operatorname{Adv}_{\mathrm{S}, \mathrm{D}, \mathrm{H}}^{\mathrm{uce}}(\lambda) & =\operatorname{Pr}\left[\operatorname{UCE}_{\mathrm{H}}^{\mathrm{S}, \mathrm{D}}(\lambda) \mid b=1\right]+\operatorname{Pr}\left[\operatorname{UCE}_{\mathrm{H}}^{\mathrm{S}, \mathrm{D}}(\lambda) \mid b=0\right]-1 \\
& =\operatorname{Pr}\left[\operatorname{Game}_{1}^{\mathrm{S}, \mathrm{D}}(\lambda)\right]-\operatorname{Pr}\left[\operatorname{Game}_{5}^{\mathrm{S}, \mathrm{D}}(\lambda)\right] \\
& \leq \sum_{i=1}^{4}\left|\operatorname{Pr}\left[\operatorname{Game}_{i}^{\mathrm{S}, \mathrm{D}}(\lambda)\right]-\operatorname{Pr}\left[\operatorname{Game}_{i+1}^{\mathrm{S}, \mathrm{D}}(\lambda)\right]\right|
\end{aligned}
$$

It remains to show that the individual games are negligibly close.

$\mathrm{Game}_{1}(\lambda)$ TO Game $2(\lambda)$. In order to reduce to the security of the indistinguishability obfuscator iO, we show that, by construction, the circuits $C_{1}[k]$ and $C_{2}\left[k^{*}, p, y^{*}\right]$ compute the same function. If $p(x)=\perp$, then $C_{2}\left[k^{*}, p, y^{*}\right]$ returns $G$.Eval $\left(k^{*}, x\right)$. If $p(x)=1$, then $x=x^{*}$ and $C_{2}\left[k^{*}, p, y^{*}\right]$ returns $y^{*}=G \cdot \operatorname{Eval}\left(k, x^{*}\right)=G \cdot \operatorname{Eval}(k, x)$. Hence, on all inputs $x, C_{2}\left[k^{*}, p, y^{*}\right]$ returns $G$.Eval $(k, x)$ and so does $C_{1}[k]$. Having established that circuits $C_{1}[k]$ and $C_{2}\left[k^{*}, p, y^{*}\right]$ compute the same functionality, $\mathrm{iO}\left(C_{1}[k]\right)$ and $\mathrm{iO}\left(C_{2}\left[k^{*}, p, y^{*}\right]\right)$ are indistinguishable and we can bound the difference between games $\mathrm{Game}_{1}$ and Game 2 by the distinguishing advantage against the indistinguishability obfuscator iO. We now formalize this intuition.

Firstly, let us externalize some of the variables that the games use and introduce a unified notation for $\mathrm{Game}_{1}$ and $\mathrm{Game}_{2}$. For $i \in\{1,2\}$, let $\mathrm{Game}_{i}\left[k, r, y^{*}, k^{*}\right](\lambda)$ be equal to the game Game $(\lambda)$ where key $k$ is chosen as PRF key, source $\mathrm{S}$ uses randomness $r$, which defines its single query to HasH, the result to that query is $y^{*}$ and the punctured key is chosen as $k^{*}$ (if such a punctured key exists, namely only in $\mathrm{Game}_{2}$ ). Note that the query $x^{*}$ of the source is well-defined by its randomness. We define $\mathcal{A}\left[k, r, y^{*}, k^{*}\right](C)$ to be an adversary against the indistinguishability obfuscator iO that gets a circuit $C$ as input, where $C$ is either an obfuscation of circuit $C_{1}[k]$ or an obfuscation of circuit $C_{2}\left[k^{*}, p, y^{*}\right]$, where $p$ is a point obfuscation of the point generated by $\mathrm{S}$ with randomness $r$. Adversary $\mathcal{A}\left[k, r, y^{*}, k^{*}\right](C)$ runs source $\mathrm{S}(r)$ to get the query $x^{*}$, returns $y^{*}$ on the single Hash query and receives leakage $L$. It then runs distinguisher $\mathrm{D}$ on input $\left(1^{\lambda}, C, L\right)$ and outputs whatever $\mathrm{D}$ outputs. We present the pseudo-code of the adversary in Figure 5 ;

If $C=C_{1}[k]$ then adversary $\mathcal{A}\left[k, r, y^{*}, k^{*}\right](C)$ perfectly simulates game Game ${ }_{1}\left[k, r, y^{*}\right](\lambda)$ and if $C=C_{2}\left[k^{*}, p, y^{*}\right]$ then the adversary simulates $\operatorname{Game}_{2}\left[k, r, y^{*}, k^{*}\right](\lambda)$. Thus, we can rewrite the difference between the two games' distributions

$$
\operatorname{Pr}\left[\operatorname{Game}_{1}(\lambda)\right]-\operatorname{Pr}\left[\operatorname{Game}_{2}(\lambda)\right]
$$

as

$$
\begin{aligned}
& \mathbb{E}_{k, r, y^{*}}\left[\operatorname{Pr}\left[\operatorname{Game}_{1}\left[k, r, y^{*}\right](\lambda)\right]\right]-\mathbb{E}_{k, r, y^{*}, k^{*}}\left[\operatorname{Pr}\left[\operatorname{Game}_{2}\left[k, r, y^{*}, k^{*}\right](\lambda)\right]\right] \\
& =\mathbb{E}_{k, r, y^{*}, k^{*}}\left[\operatorname{Pr}\left[\operatorname{Game}_{1}\left[k, r, y^{*}\right](\lambda)\right]-\operatorname{Pr}\left[\operatorname{Game}_{2}\left[k, r, y^{*}, k^{*}\right](\lambda)\right]\right] \\
& =\mathbb{E}_{k, r, y^{*}, k^{*}}\left[\operatorname{Pr}\left[\mathcal{A}\left[k, r, y^{*}, k^{*}\right]\left(1^{\lambda}, \mathrm{iO}\left(C_{1}[k]\right)\right)=1\right]-\operatorname{Pr}\left[\mathcal{A}\left[k, r, y^{*}, k^{*}\right]\left(1^{\lambda}, \mathrm{iO}\left(C_{2}\left[k^{*}, p, y^{*}\right]\right)\right)=1\right]\right] \\
& =\mathbb{E}_{k, r, y^{*}, k^{*}}\left[\operatorname{Adv}_{\mathrm{iO}, \mathcal{A}\left[k, r, y^{*}, k^{*}\right], C_{1}[k], C_{2}\left[k^{*}, p, y^{*}\right]}^{\text {io }}(\lambda)\right] \\
& \leq \max _{k, r, y^{*}, k^{*}} \operatorname{Adv}_{\mathrm{iO}, \mathcal{A}\left[k, r, y^{*}, k^{*}\right], C_{1}[k], C_{2}\left[k^{*}, p, y^{*}\right]}^{\text {io }}(\lambda)
\end{aligned}
$$




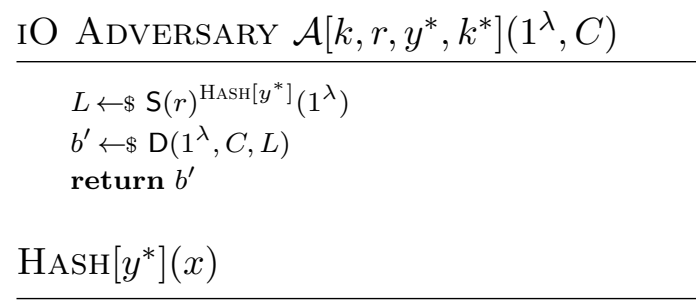

return $y^{*}$

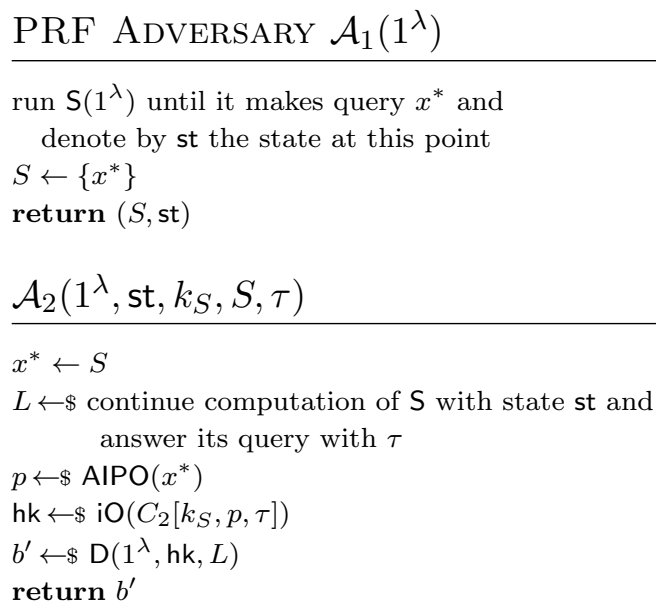

Figure 5: Pseudo-code of the $\mathrm{iO}$ adversary used in game transition from Game ${ }_{1}$ to Game $\mathrm{G}_{2}$ on the left, and the puncturable $\mathrm{PRF}$ adversary used in the transition of $\mathrm{Game}_{2}$ to $\mathrm{Game}_{3}$ on the right.

By the security of the indistinguishability obfuscator, the advantage of any efficient adversary is negligible and, hence, also $\max _{k, r, y^{*}, k^{*}} \operatorname{Adv}_{\mathrm{iO}, \mathcal{A}\left[k, r, y^{*}, k^{*}\right], C_{1}[k], C_{2}\left[k^{*}, p, y^{*}\right]}(\lambda)$ is negligible.

$\mathrm{Game}_{2}(\lambda)$ то Game $(\lambda)$. We reduce the difference between $\mathrm{Game}_{2}$ and Game 3 to the security of the puncturable PRF $G$. We define an adversary $\left(\mathcal{A}_{1}, \mathcal{A}_{2}\right)$ against the puncturable PRF as follows. On input the security parameter, adversary $\mathcal{A}_{1}$ runs source $\mathrm{S}\left(1^{\lambda}\right)$ on the security parameter. When source $\mathrm{S}$ makes its single HASH query $x^{*}$, adversary $\mathcal{A}_{1}$ stops, outputs $\left\{x^{*}\right\}$ as puncture set $S$ together with the current state st of the source $\mathrm{S}$. Adversary $\mathcal{A}_{2}$ gets as input state st, the punctured key $k^{*}=k_{S}$, the puncture point $\left\{x^{*}\right\}=S$ and a target value $\tau$ which is either $G$. Eval $\left(k, x^{*}\right)$ or a uniformly random value. Adversary $\mathcal{A}_{2}$ uses the source's state st to continue the simulation of the source $\mathrm{S}$, which expects an answer from its HAsH oracle. The adversary $\mathcal{A}_{2}$ passes value $\tau$ to $\mathrm{S}$ and receives leakage $L$. It then constructs an obfuscation $p \leftarrow \$ \operatorname{AIPO}\left(p_{x^{*}}\right)$ as well as an obfuscation hk $\leftarrow \$ \mathrm{iO}\left(C_{2}\left[k^{*}, p, \tau\right]\right)$. Subsequently, it runs distinguisher D on input $\left(1^{\lambda}, \mathrm{hk}, L\right)$ and outputs whatever D outputs. We give the pseudo-code of the adversary in Figure 5 .

If $\tau=G$. Eval $\left(k, x^{*}\right)$, then adversary $\left(\mathcal{A}_{1}, \mathcal{A}_{2}\right)$ perfectly simulates $G_{a m e}$ and otherwise it perfectly simulates Game3. Thus, we have that

$$
\operatorname{Pr}\left[\operatorname{Game}_{2}(\lambda)\right]-\operatorname{Pr}\left[\operatorname{Game}_{3}(\lambda)\right] \leq \operatorname{Adv}_{G, \mathcal{A}_{1}, \mathcal{A}_{2}}^{\mathrm{p}-\mathrm{pr}}(\lambda)
$$

which by the security of the puncturable PRF $G$ is negligible.

$\mathrm{Game}_{3}(\lambda)$ TO $\mathrm{Game}_{4}(\lambda)$. We show that the circuits $C_{2}\left[k^{*}, p, y^{*}\right]$ and $C_{3}\left[k, p, y^{*}\right]$ compute the same function. As the functionality of punctured PRFs is preserved under puncturing, for all $x$ where $p(x)=\perp$, it holds that $C_{2}\left[k^{*}, p, y^{*}\right](x)=G$.Eval $\left(k^{*}, x\right)=G$.Eval $(k, x)=C_{3}\left[k, p, y^{*}\right]$. For $x$ with $p(x)=1$, by definition, both circuits $C_{2}\left[k^{*}, p, y^{*}\right](x)$ and $C_{3}\left[k, p, y^{*}\right]$ return $y^{*}$.

As both circuits are equal, $\mathrm{iO}\left(C_{2}\left[k^{*}, p, y^{*}\right]\right)$ and $\mathrm{iO}\left(C_{3}\left[k, p, y^{*}\right]\right)$ are indistinguishable by the security of indistinguishability obfuscation. Analogously to the first game hop, we get that

$$
\operatorname{Pr}\left[\operatorname{Game}_{3}(\lambda)\right]-\operatorname{Pr}\left[\operatorname{Game}_{4}(\lambda)\right] \leq \max _{k, x^{*}, y^{*}, k^{*}} \operatorname{Adv}_{\mathrm{iO}, \mathcal{A}\left[k, x^{*}, y^{*}, k^{*}\right], C_{2}\left[k^{*}, p, y^{*}\right], C_{3}\left[k, p, y^{*}\right]}(\lambda) .
$$




\begin{tabular}{l}
$\frac{\mathrm{DIO} \text { SAMPLER Sam }\left(1^{\lambda}\right)}{x^{*}, y^{*} \leftarrow \perp}$ \\
$k \leftarrow \$ G \cdot \mathrm{KGen}\left(1^{\lambda}\right)$ \\
$L \leftarrow \$ \mathrm{~S}^{\mathrm{HASH}}\left(1^{\lambda}\right)$ \\
$p \leftarrow \$ \operatorname{AIPO}\left(x^{*}\right)$ \\
$\operatorname{return}\left(C_{3}\left[k, p, y^{*}\right], C_{4}[k], L\right)$ \\
$\operatorname{HASH}(x)$ \\
\hline$x^{*} \leftarrow x$ \\
$y^{*} \leftarrow \$\{0,1\}^{\mathrm{H} . o l}(\lambda)$ \\
return $y^{*}$
\end{tabular}

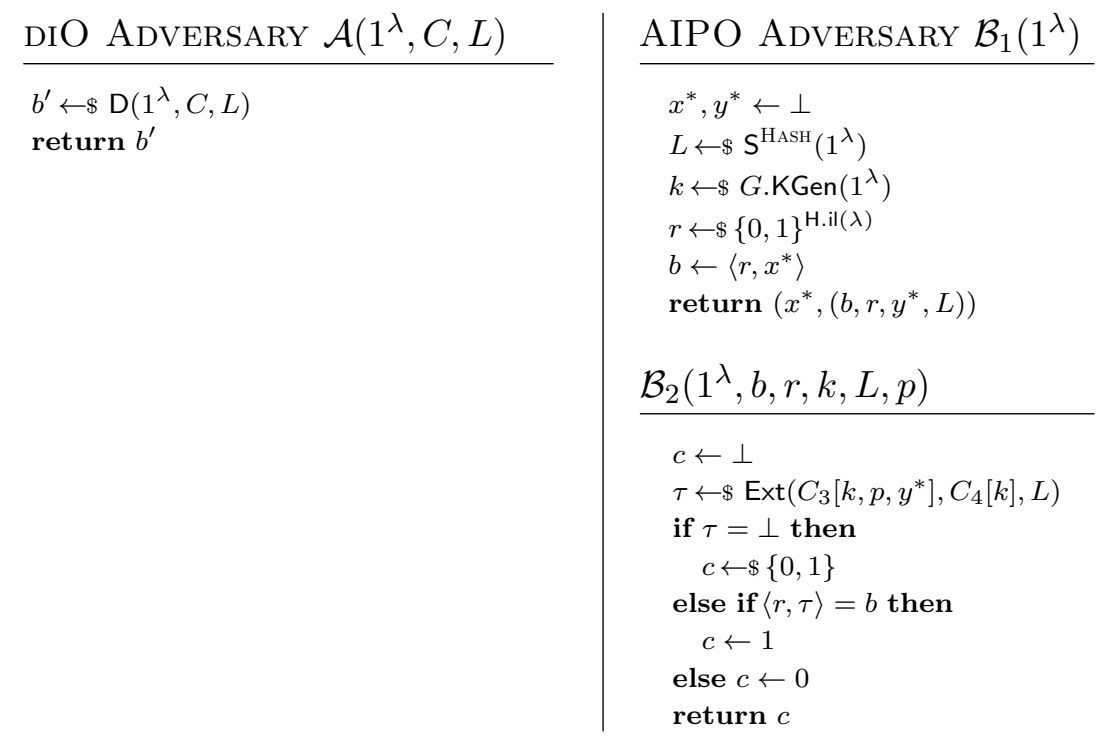

Figure 6: On the left, pseudo-code of the differing-inputs sampler Sam that we use in the transition from Game ${ }_{3}$ to Game $_{4}$. We present the differing-inputs adversary $\mathcal{A}$ in the middle and the AIPO adversary $\left(\mathcal{B}_{1}, \mathcal{B}_{2}\right)$ on the right. Both of them are used in the game transition from $\mathrm{Game}_{4}$ to $\mathrm{Game}_{5}$. Note that the HASH oracle given to $\mathrm{S}$ in the description of $\mathcal{B}_{1}$ is equivalent to the HASH oracle used by sampler Sam on the left.

$\mathrm{Game}_{4}(\lambda)$ TO Game $(\lambda)$. By construction, the circuits $C_{3}\left[k, p, y^{*}\right]$ and $C_{4}[k]$ only differ on points where $p(x)$ is not equal to $\perp$, that is, they differ on a single point, which is the query point $x^{*}$. We will bound the difference between games $\mathrm{Game}_{4}$ and $\mathrm{Game}_{5}$ by the differing-inputs security of the indistinguishability obfuscator iO. For this, we build on a result by Boyle, Chung and Pass (here given as Theorem 2.5 who show that any indistinguishability obfuscator is also a differing-inputs obfuscator for differing-inputs circuits which differ on at most polynomially many points [BCP14]. As explained above, our circuits differ only on a single point and we can, thus, apply their theorem. In order to argue with the security property of differing-inputs obfuscation, we need to show that the family of circuit pairs $\left(C_{3}\left[k, p, y^{*}\right], C_{4}[k]\right.$, Sam $)$ is differing-inputs, where Sam is the circuit sampler that runs the same steps as game Game ${ }_{4}$ up-to and including the obfuscation of the point function $p_{x^{*}}$, constructs circuits $C_{3}\left[k, p, y^{*}\right]$ and $C_{4}[k]$, and outputs $\left(C_{3}\left[k, p, y^{*}\right], C_{4}[k], L\right)$. We give the pseudo-code of sampler Sam in Figure 6 .

Claim 3.4. If AIPO is a secure AIPO obfuscator (see Definition 2.7), then the family of circuit pairs $\left(C_{3}\left[k, p, y^{*}\right], C_{4}[k]\right.$, Sam $)$ is differing-inputs.

Before proving Claim 3.4, we show how to use it to prove that the difference between $\operatorname{Game}_{4}(\lambda)$ and $\operatorname{Game}_{5}(\lambda)$ is small. Theorem 2.5 by Boyle et al. [BCP14] says that, if a family is differing-inputs and only differs on at most polynomially many points, then their indistinguishability obfuscations are indistinguishable. Claim 3.4 establishes that the family $\left(C_{3}\left[k, p, y^{*}\right], C_{4}[k]\right.$, Sam $)$ is differing-inputs, and we already observed that circuits $C_{3}\left[k, p, y^{*}\right]$ and $C_{4}[k]$ only differ on a single input value. Hence, Theorem 2.5 allows us to do an analysis similar to the one from the first game hop. That is, we define adversary $\mathcal{A}$ which gets as input a circuit $C$ and leakage $L$ where $C$ is either an indistinguishability obfuscation of circuit $C_{3}\left[k, p, y^{*}\right]$ or of circuit $C_{4}[k]$ as sampled by Sam. It runs distinguisher $\mathrm{D}$ on input $\left(1^{\lambda}, C, L\right)$ and outputs whatever $\mathrm{D}$ outputs. We give the pseudo-code of adversary $\mathcal{A}$ in Figure 6 .

If $C=\mathrm{iO}\left(C_{3}\left[k, p, y^{*}\right]\right)$, then adversary $\mathcal{A}$ perfectly simulates $\operatorname{Game}_{4}(\lambda)$, and if $C=\mathrm{iO}\left(C_{4}[k]\right)$, then it perfectly simulates $\operatorname{Game}_{5}(\lambda)$. Thus, we have

$$
\operatorname{Pr}\left[\operatorname{Game}_{4}(\lambda)\right]-\operatorname{Pr}\left[\operatorname{Game}_{5}(\lambda)\right] \leq \operatorname{Adv}_{\mathrm{iO}, \mathcal{A}, C_{3}, C_{4}, \operatorname{Sam}}^{\mathrm{dio}}(\lambda) \leq \operatorname{negl}(\lambda)
$$


We now proceed to proving Claim 3.4. Assume there exists an adversary (i.e., an extractor) Ext against the differing-inputs of the above circuit family which receives as input $\left(C_{3}\left[k, p, y^{*}\right], C_{4}[k], L\right)$ and outputs a value $\tau$ such that $C_{3}\left[k, p, y^{*}\right](\tau) \neq C_{4}[k](\tau)$. Then, $p(\tau)=1$ and thus, intuitively, Ext breaks the AIPO property of the point obfuscation scheme. Let us now make this intuition formal.

We construct adversary $\left(\mathcal{B}_{1}, \mathcal{B}_{2}\right)$ where $\mathcal{B}_{1}$ describes an unpredictable distribution. On input the security parameter, $\mathcal{B}_{1}$ runs source $\mathrm{S}\left(1^{\lambda}\right)$ and answers its single HASH query $x^{*}$ with a uniformly random value $y^{*}$ and then receives leakage $L$ from $\mathrm{S}$. $\mathcal{B}_{1}$ draws a random string $r$. It then computes $b:=\left\langle r, x^{*}\right\rangle$ and finally outputs $\left(x^{*},\left(b, r, y^{*}, L\right)\right)$.

Adversary $\mathcal{B}_{2}$ gets as input the security parameter, the auxiliary input $\left(b, r, y^{*}, L\right)$ and an obfuscation $p$ which is either an obfuscation of point function $p_{x^{*}}$ or of a point function $p_{u}$ for a uniformly random $u$. It samples a random key $k \leftarrow \$ G . \operatorname{KGen}(\lambda)$ and constructs circuits $C_{3}\left[k, p, y^{*}\right]$ and $C_{4}[k]$. It then calls Ext on input $\left(C_{3}\left[k, p, y^{*}\right], C_{4}[k], L\right)$ to receive a value $\tau$. If Ext outputs $\tau=\perp$, then $\mathcal{B}_{2}$ flips a bit and returns the outcome of the bitflip. Else, if $\tau$ is such that $C_{3}\left[k, p, y^{*}\right](\tau) \neq C_{4}[k](\tau)$ and hence $p(\tau)=1$ then $\mathcal{B}_{2}$ outputs 1 if $\langle r, \tau\rangle$ equals $b$ and 0 otherwise.

If $p$ is an obfuscation of $p_{x^{*}}$ then circuits $C_{3}\left[k, p, y^{*}\right]$ and $C_{4}[k]$ differ on input $\tau$ if and only if $\tau=x^{*}$, unless $y^{*}=G$.Eval $\left(k, x^{*}\right)$, which happens only with negligible probability. Hence, if the differing-inputs adversary Ext outputs $\tau$, then $\tau=x^{*}$ and, thus, with probability $1, \mathcal{B}_{2}$ will output 1 . If, on the other hand, $p$ is an obfuscation of $p_{u}$, then the circuits $C_{3}\left[k, p, y^{*}\right]$ and $C_{4}[k]$ differ on input $\tau$ if and only if $\tau=u$. Hence, if the differing-inputs adversary Ext outputs $\tau$, then $\tau=u$ and thus, $\mathcal{B}$ will only output 1 with probability $\frac{1}{2}\left(\right.$ since $\left.\operatorname{Pr}[\langle u, r\rangle=b]=\frac{1}{2}\right)$. Let us make the probability analysis formal. Let $d=0$ describe the event that in the AIPO-game, $p_{x^{*}}$ gets obfuscated, and $d=1$ describe the event that in the AIPO-game, $p_{u}$ gets obfuscated for a random $u$. Let $\epsilon$ be the probability that Ext returns a value $\tau \neq \perp$ in the differing-inputs game, that is, $\epsilon:=\operatorname{Pr}[\perp \neq$ Ext $\mid d=0]$. Note, that for readability we do not specify the input of adversaries Ext and $\mathcal{B}_{2}$ in the following treatment. We now consider the distinguishing probability of adversary $\mathcal{B}_{2}$

$$
\operatorname{Pr}\left[\mathcal{B}_{2}=1 \mid d=0\right]-\operatorname{Pr}\left[\mathcal{B}_{2}=1 \mid d=1\right]
$$

which can be rewritten as

$$
\begin{aligned}
= & \operatorname{Pr}\left[\mathcal{B}_{2}=1 \mid d=0, \text { Ext } \neq \perp\right] \cdot \operatorname{Pr}[\text { Ext } \neq \perp \mid d=0]+ \\
& \operatorname{Pr}\left[\mathcal{B}_{2}=1 \mid d=0, \text { Ext }=\perp\right] \cdot \operatorname{Pr}[\text { Ext }=\perp \mid d=0]- \\
& \operatorname{Pr}\left[\mathcal{B}_{2}=1 \mid d=1\right] \\
= & \operatorname{Pr}[\text { Ext } \neq \perp \mid d=0]+\frac{1}{2} \cdot \operatorname{Pr}[\text { Ext }=\perp \mid d=0]-\operatorname{Pr}\left[\mathcal{B}_{2}=1 \mid d=1\right] \\
= & \operatorname{Pr}[\text { Ext } \neq \perp \mid d=0]+\frac{1}{2} \cdot(1-\operatorname{Pr}[\text { Ext } \neq \perp \mid d=0])-\operatorname{Pr}\left[\mathcal{B}_{2}=1 \mid d=1\right] \\
= & \frac{1}{2} \cdot \operatorname{Pr}[\text { Ext } \neq \perp \mid d=0]+\frac{1}{2}-\operatorname{Pr}\left[\mathcal{B}_{2}=1 \mid d=1\right]
\end{aligned}
$$

In the following, we consider the random variable $U$ to describe the underlying choice of point function $p_{u}$ (in case $d=1$ ).

$$
\begin{aligned}
& =\frac{1}{2} \epsilon+\frac{1}{2}-\operatorname{Pr}\left[\mathcal{B}_{2}=1 \mid d=1, \text { Ext } \neq \perp\right] \cdot \operatorname{Pr}[\text { Ext } \neq \perp \mid d=1]+ \\
& \qquad \operatorname{Pr}\left[\mathcal{B}_{2}=1 \mid d=1, \text { Ext }=\perp\right] \cdot \operatorname{Pr}[\text { Ext }=\perp \mid d=1] \\
& =\frac{1}{2} \epsilon+\frac{1}{2}-
\end{aligned}
$$




$$
\begin{array}{r}
\frac{1}{2^{\mathrm{H} . \mathrm{il}(\lambda)}} \sum_{u \in\{0,1\}^{\mathrm{H} . \mathrm{il}(\lambda)}}\left(\operatorname{Pr}\left[\mathcal{B}_{2}=1 \mid d=1, U=u, \text { Ext } \neq \perp\right] \cdot \operatorname{Pr}[\text { Ext } \neq \perp \mid U=u, d=1]+\right. \\
\left.\operatorname{Pr}\left[\mathcal{B}_{2}=1 \mid d=1, U=u, \text { Ext }=\perp\right] \cdot \operatorname{Pr}[\operatorname{Ext}=\perp \mid U=u, d=1]\right)
\end{array}
$$

If extractor Ext outputs a value $u$ (given that $d=1$ ), then the probability of $\mathcal{B}_{2}$ of outputting 1 , that is, $\operatorname{Pr}\left[\mathcal{B}_{2}=1 \mid d=1, U=u\right.$, Ext $\left.\neq \perp\right]$ is equivalent to $\operatorname{Pr}_{R, b}[\langle R, u\rangle=b]$ where random variable $R$ denotes the choice of value $r$ by $\mathcal{B}_{1}$ to compute $b=\left\langle r, x^{*}\right\rangle$. Note that extractor Ext is independent of $R$ and $b$ and, thus, we have that $\operatorname{Pr}_{R, b}[\langle R, u\rangle=b]=\frac{1}{2}$.

$$
\begin{aligned}
= & \frac{1}{2} \epsilon+\frac{1}{2}- \\
& \frac{1}{2^{\mathrm{H} . \mathrm{il}(\lambda)}} \sum_{u \in\{0,1\}^{\mathrm{H} \cdot \mathrm{il}(\lambda)}}\left(\operatorname{Pr}_{R, b}[\langle R, u\rangle=b] \cdot \operatorname{Pr}[\text { Ext } \neq \perp \mid U=u, d=1]+\frac{1}{2} \cdot \operatorname{Pr}[\operatorname{Ext}=\perp \mid U=u, d=1]\right) \\
= & \frac{1}{2} \epsilon+\frac{1}{2}-\frac{1}{2^{\mathrm{H} . \mathrm{il}(\lambda)}} \sum_{u \in\{0,1\}^{\mathrm{H} . \mathrm{II}(\lambda)}}\left(\frac{1}{2} \cdot(\operatorname{Pr}[\operatorname{Ext} \neq \perp \mid U=u, d=1]+\operatorname{Pr}[\operatorname{Ext}=\perp \mid U=u, d=1])\right) \\
= & \frac{1}{2} \epsilon+\frac{1}{2}-\frac{1}{2^{\mathrm{H} . \mathrm{il}(\lambda)}} \sum_{u \in\{0,1\}^{\mathrm{H} . \mathrm{II}(\lambda)}} \frac{1}{2} \cdot 1=\frac{1}{2} \epsilon
\end{aligned}
$$

To finish the proof of Claim 3.4, we need to argue that $\mathcal{B}_{1}$ implements an unpredictable distribution. By assumption, the source $S$ is strongly computationally unpredictable (i.e., $\mathbf{S} \in \mathcal{S}^{\text {s-cup }}$ ) and hence leakage $L$ hides $x^{*}$ even in the presence of $y^{*}$. Thus, to see that $\mathcal{B}_{1}$ defines an unpredictable distribution, we need to argue that $x^{*}$ remains unpredictable if additionally given a single bit of $x^{*}$. But a single bit can be guessed with probability $\frac{1}{2}$. Hence, $\left(\mathcal{B}_{1}, \mathcal{B}_{2}\right)$ breaks the security of the AIPO obfuscation, which concludes the proof of Claim 3.4 and the proof of Theorem 3.3 .

\subsection{A UCE Construction Secure Against Sources in $\mathcal{S}^{\text {s-sup }} \cap \mathcal{S}^{q \text {-query }}$}

In this section we prove that our construction is also UCE-secure with respect to sources which are strongly unpredictable in a statistical sense and which allow the source to make $q$-many queries for any polynomial $q:=q(\lambda)$. That is, we consider sources in class $\mathcal{S}^{\text {s-sup }} \cap \mathcal{S}^{q \text {-query }}$.

In case we allow the source to make $q$ many queries, the first observation is that we need to choose the size of our obfuscated circuit such we can puncture at $q$ many points. For each point, we will encode a random string into the circuit and thus, the circuit size grows with the number of points we need to puncture out. Besides this, the construction is identical to the one before with the exception that we need a different (incomparable) security property of our point function obfuscation scheme. That is, we require the point obfuscator to be a $q$-composable VGB obfuscator secure in the presence of statistically unpredictable auxiliary information which implies an AIPO obfuscator with statistically unpredictable auxiliary information (see Section 2.1).

Theorem 3.5. Let $q$ be a polynomial. If $G$ is a secure puncturable PRF, if $\mathrm{iO}$ is a secure indistinguishability obfuscator and if there exist a q-composable VGB point obfuscator for statistically unpredicatable auxiliary input, then the hash function family $\mathrm{H}$ defined in Construction [3.2 is $\mathrm{UCE}\left[\mathcal{S}^{\text {s-sup }} \cap \mathcal{S}^{q-q u e r y}\right]$ secure.

The proof follows analogously to the proof of Theorem 3.3, except for puncturing at several points instead of a single point and therefore, we reduce to t-composable VGB point obfuscation. The proof is deferred to Appendix C. For an overview of the game-hops, see Figure 9. 


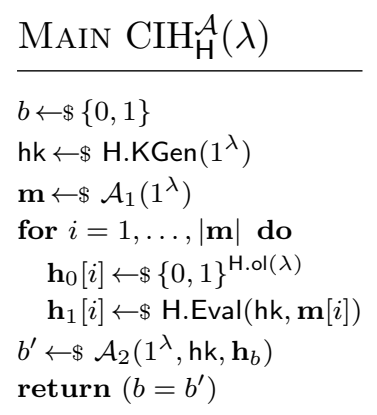

Figure 7: The security game for correlatedinput hash functions.

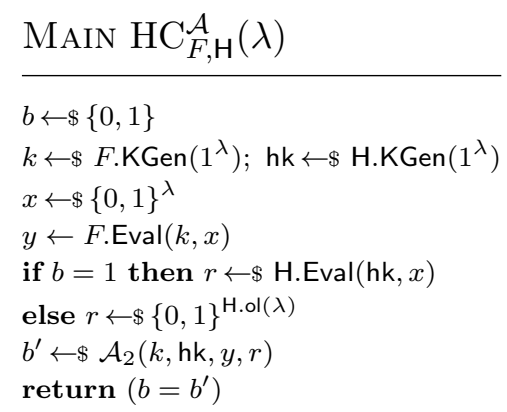

Figure 8: The security game for hardcore functions.

\section{Applications}

In the following section we describe the applications that our UCE constructions fulfill. Our UCE $\left[\mathcal{S}^{\text {s-cup }} \cap\right.$ $\mathcal{S}^{1 \text {-query }}$-secure function can be shown to be a universal hardcore function for any one-way function and our $\mathrm{UCE}\left[\mathcal{S}^{\text {s-sup }} \cap \mathcal{S}^{q-q u e r y}\right]$-secure function achieves correlated-input security. We note that our $\mathrm{UCE}\left[\mathcal{S}^{\text {s-sup }} \cap \mathcal{S}^{q \text {-query }}\right]$ construction is also sufficient to instantiate proof-of-storage schemes and we refer to $[\mathrm{BHK} 13 \mathrm{~b}]$ for further details.

\subsection{Hash Functions Secure under Correlated Inputs}

Correlated-input secure hash functions $(\mathrm{CIH})$ demand that an adversary that is able to obtain a sequence of (potentially correlated) hash values cannot distinguish between these being real or uniformly random assuming that source values come from a distribution that has super-logarithmic min-entropy. The notion was introduced by Goyal, O'Neill, and Rao GOR11 and GOR present several constructions for limited $\mathrm{CIH}$ in the standard model. However, constructions for full CIH are only known in the random oracle model, even if the number of number of queries are bounded by a polynomial. Bellare, Hoang, and Keelvedhi show that hash functions secure under $\operatorname{UCE}\left[\mathcal{S}^{\text {sup }} \cap \mathcal{S}^{\text {splt }}\right]$-assumptions are also $\mathrm{CIH}$ secure [BHK13b]. As statistically strong unpredictability is a strictly larger source class than statistically secure split sources (see Lemma 3.1) our construction from Section 3.3 yields the first candidate construction of $q$-query CIH-secure hash functions.

We present game $\mathrm{CIH}_{\mathrm{H}}^{\mathcal{A}}$ for correlated-input secure hash functions in Figure 7. We say that a function $\mathrm{H}$ is CIH-secure if the advantage of any admissible PPT adversary $\mathcal{A}=\left(\mathcal{A}_{1}, \mathcal{A}_{2}\right)$ defined as

$$
\operatorname{Adv}_{\mathrm{H}, \mathcal{A}}:=2 \cdot \operatorname{Pr}\left[\operatorname{CIH}_{\mathrm{H}}^{\mathcal{A}}(\lambda)\right]-1
$$

is negligible. An adversary is admissible if on input the security parameter adversary $\mathcal{A}_{1}$ outputs a vector $\mathbf{m}$ of distinct values and of length $|\mathbf{m}|=v(\lambda)$ where $v$ is a polynomial depending on $\mathcal{A}_{1}$. Furthermore, we require that the guessing probability of each entry is negligible, that is, the min-entropy of $[i]$ for all $i=1, \ldots, v(\lambda)$ must be at least super-logarithmic in the security parameter.

BHK give the following theorem [BHK13b]:

Theorem 4.1 ([BHK13b] $)$. If $\mathrm{H}$ is $\mathrm{UCE}\left[\mathcal{S}^{\text {sup }} \cap \mathcal{S}^{\text {splt }}\right]$-secure then $\mathrm{H}$ is a correlated-input hash function $(\mathrm{CIH})$.

In our construction of a $\operatorname{UCE}\left[\mathcal{S}^{\text {s-sup }} \cap \mathcal{S}^{q-\text { query }}\right]$ the number of queries that we can allow a source to make can be regarded as a parameter of the key generation function. We give the following adaption of $\mathrm{CIH}$-secure hash functions called q-query correlated-input secure hash functions where the number of queries is specified as part of the key generation process. 
Definition 4.2. Let $q$ be a polynomial. A hash construction $\mathrm{H}$ is called q-query correlated-input secure if its key generation algorithm takes as input parameter $q$ and if the advantage of any adversary $\mathcal{A}=\left(\mathcal{A}_{1}, \mathcal{A}_{2}\right)$ where $\mathcal{A}_{1}$ outputs a message vector of length at most $q$ in the $\mathrm{CIH}_{\mathrm{H}}^{\mathcal{A}}$ game is negligible.

Combining Theorems 3.5 and 4.1 we get:

Proposition 4.3. Let $q$ be a polynomial. If $G$ is a secure puncturable PRF, if iO is a secure indistinguishability obfuscator and if there exist a q-composable VGB point obfuscator for statistically unpredicatable auxiliary input, then the hash function family defined in Construction 3.2 is q-CIH secure.

\subsection{Universal Hardcore Functions}

A hardcore function for a one-way function $f$ is a (deterministic) algorithm whose output on a random point $x$ is indistinguishable from random even given the image under $f$, that is, given $f(x)$. Random oracles are natural hardcore functions. BHK show that also UCE-secure functions, secure with respect to computationally unpredictable split sources are hardcore for any one-way function.

Let $F$ be a (possibly keyed) one-way function. We say that function $\mathrm{H}$ is hardcore for $F$ if the advantage of any PPT adversary $\mathcal{A}$ in game $\mathrm{HC}_{F, \mathrm{H}}^{\mathcal{A}}$ (given in Figure 8 is negligible, where we define the advantage as

$$
\operatorname{Adv}_{\mathrm{H}, \mathcal{A}}:=2 \cdot \operatorname{Pr}\left[\mathrm{HC}_{F, \mathrm{H}}^{\mathcal{A}}(\lambda)\right]-1 .
$$

Bellare, Hoang, and Keelvedhi show that any UCE-secure function with respect to split sources that are computationally unpredictable are universal hardcore functions. We recall their result:

Theorem 4.4 ([BHK13b] $)$. If $\mathrm{H}$ is $\mathrm{UCE}\left[\mathcal{S}^{\text {cup }} \cap \mathcal{S}^{\text {splt }} \cap \mathcal{S}^{1 \text {-query }}\right]$-secure then $\mathrm{H}$ is a hardcore function for any one-way function.

Combined with Construction 3.2 and Theorem 3.3 we get an instantiation of a universal hardcore function.

Proposition 4.5. If $G$ is a secure puncturable PRF, if $\mathrm{iO}$ is a secure indistinguishability obfuscator and if AIPO exists, then the hash function family $\mathrm{H}$ defined in Construction 3.2 is a universal hardcore function.

The BR93 PKE scheme. In their seminal paper on random oracles, Bellare and Roggaway proposed a very simple, yet elegant public-key encryption scheme [BR93] based on a single trapdoor function $f$. The public-key of the encryption scheme is set to the evaluation key of the trapdoor function, and the private key is the set to be the trapdoor. To encrypt a message $m$ the encryption algorithm chooses a random value $x$ and outputs $(f(x), \mathrm{H}(x) \oplus m)$, where $\mathrm{H}$ is a hash function. For decryption, one inverts $f(x)$ using the trapdoor and can, thus, recover message $m$. Bellare et al. show that we can safely instantiate the hash function with any $\operatorname{UCE}\left[\mathcal{S}^{\text {cup }} \cap \mathcal{S}^{\text {splt }} \cap \mathcal{S}^{1 \text {-query }}\right.$ [ BHK13b]. Combined with Construction 3.2 and Theorem 3.3 we get an instantiation of that scheme.

Proposition 4.6. If $f$ is a trapdoor one-way function, if $G$ is a secure puncturable PRF, if iO is a secure indistinguishability obfuscator and if AIPO exists, then the BR93 PKE scheme [BR93] instantiated with $f$ and the hash function family $\mathrm{H}$ defined in Construction 3.2 is IND-CPA secure. 


\section{The BFM Impossibility Result}

In a recent work, Brzuska, Farshim, and Mittelbach [BFM14] show that assuming indistinguishability obfuscation exists, no standard model hash construction can be $\mathrm{UCE}\left[\mathcal{S}^{\text {cup }}\right]$-secure, that is, UCE secure with respect to computationally unpredictable sources. In the following section we discuss the possibility of the BFM attack being extended to also cover UCE with respect to strongly unpredictable sources and implications thereof.

The BFM atTack. Let us recall the intuition behind the BFM attack. Consider the source $\mathrm{S}_{\mathrm{BFM}}$ that makes a single (random) query $x$ to receive $\operatorname{HASH}(x)$. It then prepares an indistinguishability obfuscation of the circuit $(\mathrm{H} . \operatorname{Eval}(\cdot, x)=y)$, that is the predicate that on input a hash key hk tests, if $\mathrm{H}$.Eval(hk, $x)=y$. If $y$ is chosen uniformly at random and if the output length of the hash function is (much) larger than the key-space, then the probability that there exists some key hk such that H.Eval(hk, $x)=y$ becomes negligible. This means that the circuit is with high probability the constant zero circuit and, thus, an indistinguishability obfuscation of the circuit does not leak anything more than the obfuscation of the constant zero circuit. It follows that the source is (computationally) unpredictable. A distinguisher, given access to the above circuit can, however, easily distinguish by simply plugging the hash key hk that it got as input into the circuit and outputting whatever the circuit outputs. We next give the pseudocode of the BFM adversary:

\begin{tabular}{|c|c|}
\hline $\operatorname{SourCE} \operatorname{S}_{\mathrm{BFM}}\left(1^{\lambda}\right)$ & Distinguisher $\mathrm{D}_{\mathrm{BFM}}\left(1^{\lambda}, \mathrm{hk}, L\right)$ \\
\hline$x \leftarrow \$\{0,1\}^{\mathrm{H.il}(\lambda)}$ & $\tilde{C} \leftarrow L$ \\
\hline$y \leftarrow \operatorname{HASH}(x)$ & $b^{\prime} \leftarrow \tilde{C}(\mathrm{hk})$ \\
\hline $\begin{array}{l}C \leftarrow(\operatorname{H.Eval}(\cdot, x)=y) \\
\tilde{C} \leftarrow \$ \mathrm{iO}(C)\end{array}$ & return $b^{\prime}$ \\
\hline
\end{tabular}

In the above intuition we relied upon the output length of the hash function being significantly larger than the key-size. To bootstrap their attack to hash functions for which this does not hold BFM simply extended the source to make multiple queries until the combined length of the received hash values is sufficiently longer than the size of the key. Bellare, Hoang, and Keelvedhi [BHK13c point out that the attack can also be extended by applying a pseudorandom generator to the output of the hash construction. The idea here is, that if $\mathrm{H}$ is a $\operatorname{UCE}\left[\mathcal{S}^{\text {cup }}\right]$-secure function then so is $\mathrm{H}^{\prime}(\cdot, \cdot):=\operatorname{PRG}(\mathrm{H}(\cdot, \cdot))$ where PRG is a pseudorandom generator.

Implausibility of EXTENDING BFM. While it is straight forward to prove that if $\mathrm{H}$ is a $\mathrm{UCE}\left[\mathcal{S}^{\text {cup }}\right]$ secure function then so is $\mathrm{H}^{\prime}(\cdot, \cdot):=\operatorname{PRG}(\mathrm{H}(\cdot, \cdot))$ this is not the case if we restrict our sources to be strongly unpredictable, that is, to source class $\mathcal{S}^{\text {s-cup }}$. The reason is that in the reduction from a predictor to the PRG security the predictor requires the single oracle answer $y$ as additional input which in the reduction would correspond to the seed of the PRG value. This, however, means that the reduction given either an image under the PRG or a uniformly random value must be able to compute the corresponding seed (if it exists) thereby breaking the PRG security on its own.

Similarly, using multiple queries seems not to allow extending the BFM attack to break our construction. In our construction the key is an obfuscation of a puncturable PRF. In order to use the puncturing technique the size of this circuit must be chosen according to the number of potential puncture points. Thus, the key size of our construction will always be strictly larger than the combined output length that can be achieved using the allowed number of queries. 
Implications of An EXTEnded ATTACK. Finally, we want to discuss the implications of a successful extension of the BFM attacks. In this case we would have the following implications:

1. iO $\Longrightarrow \neg \mathrm{UCE}\left[\mathcal{S}^{\text {s-cup }}\right]$

2. $\mathrm{iO}+\mathrm{AIPO} \Longrightarrow \mathrm{UCE}\left[\mathcal{S}^{\text {s-cup }}\right]$

Combining the two would result in the statement

$$
\mathrm{iO} \Longrightarrow \neg \mathrm{AIPO}
$$

that is, if indistinguishability obfuscation exists then point function obfuscation secure in the presence of auxiliary inputs does not exist and vice versa. This would be a surprising result as currently we hope that both forms of obfuscation exists. While iO has been used in numerous works lately [SW14, BCP14, $\mathrm{ABG}^{+} 13$, GGHR14, HSW14, BZ14, BST14, point function obfuscation secure in the presence of auxilliary inputs has been used, for example, to circumvent black-box impossibility results and construct 3-round proofs with negligible soundness error satisfying the zero-knowledge notions weak $Z K$ and witness hiding [GK96] and very recently to construct CCA secure public key encryption schemes [MH14a]. In a very recent work Brzuska and Mittelbach [BM14] give a partial answer to this question and show that indistinguishability obfuscation and multi-bit output point function obfuscation secure in the presence of auxiliary information (MB-AIPO) are mutually exclusive. A multi-bit output point function $p_{x, m}$ is similar to a plain point function $p_{x}$ except that the the input $x$ is mapped to $m$ instead of to 1 . We note that the their techniques do not seem to carry over to plain AIPO.

An intriguing direction for further research is, thus, the study of point obfuscation with auxiliary inputs in the lights of the new results regarding indistinguishability obfuscation. Finally, let us note that in case AIPOs, indeed, do not exist that our result could be salvaged by considering a statistical version of strong unpredictability. This fix, was also proposed by BFM (and independently by BHK) to salvage a large number of applications for UCEs [BFM14, BHK13b].

\section{Acknowledgments}

We thank the Asiacrypt 2014 reviewers for the many constructive comments. We especially thank Paul Baecher, Mihir Bellare, Pooya Farshim, Victoria Fehr, Giorgia Azzurra Marson, Adam O’Neill and Daniel Wichs for many helpful comments and discussions throughout the various stages of this work. Christina Brzuska was supported by the Israel Science Foundation (grant 1076/11 and 1155/11), the Israel Ministry of Science and Technology grant 3-9094), and the German-Israeli Foundation for Scientific Research and Development (grant 1152/2011). Arno Mittelbach was supported by CASED (www.cased.de) and the German Research Foundation (DFG) SPP 1736.

\section{References}

[ABG $\left.{ }^{+} 13\right]$ Prabhanjan Ananth, Dan Boneh, Sanjam Garg, Amit Sahai, and Mark Zhandry. Differinginputs obfuscation and applications. Cryptology ePrint Archive, Report 2013/689, 2013. http://eprint.iacr.org/2013/689. (Cited on pages 3 11 and 28.)

[AGIS14] Prabhanjan Ananth, Divya Gupta, Yuval Ishai, and Amit Sahai. Optimizing obfuscation: Avoiding Barrington's theorem. Cryptology ePrint Archive, Report 2014/222, 2014. http://eprint.iacr.org/2014/222. (Cited on page 34) 
[BBO07] Mihir Bellare, Alexandra Boldyreva, and Adam O'Neill. Deterministic and efficiently searchable encryption. In Alfred Menezes, editor, Advances in Cryptology - CRYPTO 2007, volume 4622 of Lecture Notes in Computer Science, pages 535-552, Santa Barbara, CA, USA, August 19-23, 2007. Springer, Berlin, Germany. (Cited on page 9])

[BC10] Nir Bitansky and Ran Canetti. On strong simulation and composable point obfuscation. In Tal Rabin, editor, Advances in Cryptology - CRYPTO 2010, volume 6223 of Lecture Notes in Computer Science, pages 520-537, Santa Barbara, CA, USA, August 15-19, 2010. Springer, Berlin, Germany. (Cited on pages 7, 8, 10, 11, and 13)

[BCP14] Elette Boyle, Kai-Min Chung, and Rafael Pass. On extractability obfuscation. In Yehuda Lindell, editor, TCC 2014: 11th Theory of Cryptography Conference, volume 8349 of Lecture Notes in Computer Science, pages 52-73, San Diego, CA, USA, February 24-26, 2014. Springer, Berlin, Germany. (Cited on pages 3, $11,12,19,22,28,34$, and 37)

[Bel14] Mihir Bellare. Personal communication. Oct, 2014. (Cited on page 17)

[BFM14] Christina Brzuska, Pooya Farshim, and Arno Mittelbach. Indistinguishability obfuscation and UCEs: The case of computationally unpredictable sources. In Juan A. Garay and Rosario Gennaro, editors, Advances in Cryptology - CRYPTO 2014, Part I, volume 8616 of Lecture Notes in Computer Science, pages 188-205, Santa Barbara, CA, USA, August 17-21, 2014. Springer, Berlin, Germany. (Cited on pages 5, 6, 8, 15, 27, and 28)

[BFO08] Alexandra Boldyreva, Serge Fehr, and Adam O'Neill. On notions of security for deterministic encryption, and efficient constructions without random oracles. In David Wagner, editor, Advances in Cryptology - CRYPTO 2008, volume 5157 of Lecture Notes in Computer Science, pages 335-359, Santa Barbara, CA, USA, August 17-21, 2008. Springer, Berlin, Germany. (Cited on page 9)

[BFOR08] Mihir Bellare, Marc Fischlin, Adam O’Neill, and Thomas Ristenpart. Deterministic encryption: Definitional equivalences and constructions without random oracles. In David Wagner, editor, Advances in Cryptology - CRYPTO 2008, volume 5157 of Lecture Notes in Computer Science, pages 360-378, Santa Barbara, CA, USA, August 17-21, 2008. Springer, Berlin, Germany. (Cited on page 9)

$\left[\mathrm{BGI}^{+} 01\right] \quad$ Boaz Barak, Oded Goldreich, Russell Impagliazzo, Steven Rudich, Amit Sahai, Salil P. Vadhan, and Ke Yang. On the (im)possibility of obfuscating programs. In Joe Kilian, editor, Advances in Cryptology - CRYPTO 2001, volume 2139 of Lecture Notes in Computer Science, pages 1-18, Santa Barbara, CA, USA, August 19-23, 2001. Springer, Berlin, Germany. (Cited on pages $3,8,10$ and 11)

[BGI $\left.{ }^{+} 12\right] \quad$ Boaz Barak, Oded Goldreich, Russell Impagliazzo, Steven Rudich, Amit Sahai, Salil Vadhan, and Ke Yang. On the (im)possibility of obfuscating programs. J. ACM, 59(2):6:16:48, May 2012. (Cited on pages 3,8 and 10.)

[BGI14] Elette Boyle, Shafi Goldwasser, and Ioana Ivan. Functional signatures and pseudorandom functions. In Hugo Krawczyk, editor, PKC 2014: 17th International Conference on Theory and Practice of Public Key Cryptography, volume 8383 of Lecture Notes in Computer Science, pages 501-519, Buenos Aires, Argentina, March 26-28, 2014. Springer, Berlin, Germany. (Cited on pages 6, 15 and 35 ) 
$\left[\mathrm{BGK}^{+} 14\right] \quad$ Boaz Barak, Sanjam Garg, Yael Tauman Kalai, Omer Paneth, and Amit Sahai. Protecting obfuscation against algebraic attacks. In Phong Q. Nguyen and Elisabeth Oswald, editors, Advances in Cryptology - EUROCRYPT 2014, volume 8441 of Lecture Notes in Computer Science, pages 221-238, Copenhagen, Denmark, May 11-15, 2014. Springer, Berlin, Germany. (Cited on page 34 )

[BHK13a] Mihir Bellare, Viet Tung Hoang, and Sriram Keelveedhi. Instantiating random oracles via UCEs. In Ran Canetti and Juan A. Garay, editors, Advances in Cryptology CRYPTO 2013, Part II, volume 8043 of Lecture Notes in Computer Science, pages 398-415, Santa Barbara, CA, USA, August 18-22, 2013. Springer, Berlin, Germany. (Cited on pages $5,13,14$ and 15 )

[BHK13b] Mihir Bellare, Viet Tung Hoang, and Sriram Keelveedhi. Instantiating random oracles via UCEs. Cryptology ePrint Archive, Report 2013/424, 2013. http://eprint.iacr org/2013/424. (Cited on pages $5,6,15,16,18,25,26$, and 28,

[BHK13c] Mihir Bellare, Viet Tung Hoang, and Sriram Keelveedhi. Personal communication. Sep, 2013. (Cited on pages 8 and 27.)

[BM14] Christina Brzuska and Arno Mittelbach. Indistinguishability obfuscation versus multibit point obfuscation with auxiliary input. In Palash Sarkar and Tetsu Iwata, editors, Advances in Cryptology - ASIACRYPT 2014, Lecture Notes in Computer Science, pages ??-??, Kaohsiung, Taiwan, December 7-11, 2014. Springer, Berlin, Germany. (Cited on pages 7,8 and 28)

[BP12] Nir Bitansky and Omer Paneth. Point obfuscation and 3-round zero-knowledge. In Ronald Cramer, editor, TCC 2012: 9th Theory of Cryptography Conference, volume 7194 of Lecture Notes in Computer Science, pages 190-208, Taormina, Sicily, Italy, March 19-21, 2012. Springer, Berlin, Germany. (Cited on pages 3, 7, 12,34 and 35)

[BP13] Elette Boyle and Rafael Pass. Limits of extractability assumptions with distributional auxiliary input. Cryptology ePrint Archive, Report 2013/703, 2013. http://eprint. iacr.org/2013/703. (Cited on page 11)

[BR93] Mihir Bellare and Phillip Rogaway. Random oracles are practical: A paradigm for designing efficient protocols. In V. Ashby, editor, ACM CCS 93: 1st Conference on Computer and Communications Security, pages 62-73, Fairfax, Virginia, USA, November 3 5, 1993. ACM Press. (Cited on page 26.)

[BR14] Zvika Brakerski and Guy N. Rothblum. Virtual black-box obfuscation for all circuits via generic graded encoding. In Yehuda Lindell, editor, TCC 2014: 11th Theory of Cryptography Conference, volume 8349 of Lecture Notes in Computer Science, pages 1-25, San Diego, CA, USA, February 24-26, 2014. Springer, Berlin, Germany. (Cited on page 34)

[BST14] Mihir Bellare, Igors Stepanovs, and Stefano Tessaro. Poly-many hardcore bits for any one-way function. In Palash Sarkar and Tetsu Iwata, editors, Advances in Cryptology ASIACRYPT 2014, Lecture Notes in Computer Science, pages ??-??, Kaohsiung, Taiwan, December 7-11, 2014. Springer, Berlin, Germany. (Cited on pages 3, 4, 6, 14, and 28)

[BW13] Dan Boneh and Brent Waters. Constrained pseudorandom functions and their applications. In Kazue Sako and Palash Sarkar, editors, Advances in Cryptology - ASIACRYPT 2013, 
Part II, volume 8270 of Lecture Notes in Computer Science, pages 280-300, Bengalore, India, December 1-5, 2013. Springer, Berlin, Germany. (Cited on pages 6, 15, and 35 )

[BZ14] Dan Boneh and Mark Zhandry. Multiparty key exchange, efficient traitor tracing, and more from indistinguishability obfuscation. In Juan A. Garay and Rosario Gennaro, editors, Advances in Cryptology - CRYPTO 2014, Part I, volume 8616 of Lecture Notes in Computer Science, pages 480-499, Santa Barbara, CA, USA, August 17-21, 2014. Springer, Berlin, Germany. (Cited on pages 3,6 and 28)

[Can97] Ran Canetti. Towards realizing random oracles: Hash functions that hide all partial information. In Burton S. Kaliski Jr., editor, Advances in Cryptology - CRYPTO'97, volume 1294 of Lecture Notes in Computer Science, pages 455-469, Santa Barbara, CA, USA, August 17-21, 1997. Springer, Berlin, Germany. (Cited on pages 3, 7, $8,12,13,34$ and 35.)

[CD08] Ran Canetti and Ronny Ramzi Dakdouk. Obfuscating point functions with multibit output. In Nigel P. Smart, editor, Advances in Cryptology - EUROCRYPT 2008, volume 4965 of Lecture Notes in Computer Science, pages 489-508, Istanbul, Turkey, April 13-17, 2008. Springer, Berlin, Germany. (Cited on pages 7 and 8)

[CGH98] Ran Canetti, Oded Goldreich, and Shai Halevi. The random oracle methodology, revisited (preliminary version). In 30th Annual ACM Symposium on Theory of Computing, pages 209-218, Dallas, Texas, USA, May 23-26, 1998. ACM Press. (Cited on page 13)

$\left[\mathrm{FGK}^{+}\right.$13] David Mandell Freeman, Oded Goldreich, Eike Kiltz, Alon Rosen, and Gil Segev. More constructions of lossy and correlation-secure trapdoor functions. Journal of Cryptology, 26(1):39-74, January 2013. (Cited on page 9)

[FOR12] Benjamin Fuller, Adam O'Neill, and Leonid Reyzin. A unified approach to deterministic encryption: New constructions and a connection to computational entropy. In Ronald Cramer, editor, TCC 2012: 9th Theory of Cryptography Conference, volume 7194 of Lecture Notes in Computer Science, pages 582-599, Taormina, Sicily, Italy, March 19-21, 2012. Springer, Berlin, Germany. (Cited on page 9)

$\left[\mathrm{GGG}^{+} 14\right]$ Shafi Goldwasser, S. Dov Gordon, Vipul Goyal, Abhishek Jain, Jonathan Katz, Feng-Hao Liu, Amit Sahai, Elaine Shi, and Hong-Sheng Zhou. Multi-input functional encryption. In Phong Q. Nguyen and Elisabeth Oswald, editors, Advances in Cryptology - EUROCRYPT 2014, volume 8441 of Lecture Notes in Computer Science, pages 578-602, Copenhagen, Denmark, May 11-15, 2014. Springer, Berlin, Germany. (Cited on page 3)

$\left[\mathrm{GGH}^{+} 13\right]$ Sanjam Garg, Craig Gentry, Shai Halevi, Mariana Raykova, Amit Sahai, and Brent Waters. Candidate indistinguishability obfuscation and functional encryption for all circuits. In 54th Annual Symposium on Foundations of Computer Science, pages 40-49, Berkeley, CA, USA, October 26-29, 2013. IEEE Computer Society Press. (Cited on pages 3. 11. and 34)

[GGHR14] Sanjam Garg, Craig Gentry, Shai Halevi, and Mariana Raykova. Two-round secure MPC from indistinguishability obfuscation. In Yehuda Lindell, editor, TCC 2014: 11th Theory of Cryptography Conference, volume 8349 of Lecture Notes in Computer Science, pages 74-94, San Diego, CA, USA, February 24-26, 2014. Springer, Berlin, Germany. (Cited on pages 3 and 28 ) 
[GGHW14a] Sanjam Garg, Craig Gentry, Shai Halevi, and Daniel Wichs. On the implausibility of differing-inputs obfuscation and extractable witness encryption with auxiliary input. In Juan A. Garay and Rosario Gennaro, editors, Advances in Cryptology - CRYPTO 2014, Part I, volume 8616 of Lecture Notes in Computer Science, pages 518-535, Santa Barbara, CA, USA, August 17-21, 2014. Springer, Berlin, Germany. (Cited on page4)

[GGHW14b] Sanjam Garg, Craig Gentry, Shai Halevi, and Daniel Wichs. On the implausibility of differing-inputs obfuscation and extractable witness encryption with auxiliary input. Cryptology ePrint Archive, Report 2013/860 version from 13 Jun 2014, Jun 2014. http: //eprint.iacr.org/, (Cited on page4)

[GGM84] Oded Goldreich, Shafi Goldwasser, and Silvio Micali. How to construct random functions (extended abstract). In 25th Annual Symposium on Foundations of Computer Science, pages 464-479, Singer Island, Florida, October 24-26, 1984. IEEE Computer Society Press. (Cited on page 15)

[GK96] Oded Goldreich and Hugo Krawczyk. On the composition of zero-knowledge proof systems. SIAM J. Comput., 25(1):169-192, February 1996. (Cited on page 28.)

[GK05] Shafi Goldwasser and Yael Tauman Kalai. On the impossibility of obfuscation with auxiliary input. In 46th Annual Symposium on Foundations of Computer Science, pages 553-562, Pittsburgh, PA, USA, October 23-25, 2005. IEEE Computer Society Press. (Cited on page 10)

[GLSW14] Craig Gentry, Allison Lewko, Amit Sahai, and Brent Waters. Indistinguishability obfuscation from the multilinear subgroup elimination assumption. Cryptology ePrint Archive, Report 2014/309, 2014. http://eprint.iacr.org/, (Cited on page 34)

[Gol90] Oded Goldreich. A note on computational indistinguishability. Information Processing Letters, pages 277-281, 1990. (Cited on page 35)

[GOR11] Vipul Goyal, Adam O’Neill, and Vanishree Rao. Correlated-input secure hash functions. In Yuval Ishai, editor, TCC 2011: 8th Theory of Cryptography Conference, volume 6597 of Lecture Notes in Computer Science, pages 182-200, Providence, RI, USA, March 28-30, 2011. Springer, Berlin, Germany. (Cited on pages 3 , and 25.)

[HILL99] Johan Håstad, Russell Impagliazzo, Leonid A. Levin, and Michael Luby. A pseudorandom generator from any one-way function. SIAM Journal on Computing, 28(4):1364-1396, 1999. (Cited on page 35)

[Hof14] Dennis Hofheinz. Fully secure constrained pseudorandom functions using random oracles. Cryptology ePrint Archive, Report 2014/372, 2014. http://eprint.iacr.org/. (Cited on page 6)

[HSW14] Susan Hohenberger, Amit Sahai, and Brent Waters. Replacing a random oracle: Full domain hash from indistinguishability obfuscation. In Phong Q. Nguyen and Elisabeth Oswald, editors, Advances in Cryptology - EUROCRYPT 2014, volume 8441 of Lecture Notes in Computer Science, pages 201-220, Copenhagen, Denmark, May 11-15, 2014. Springer, Berlin, Germany. (Cited on pages 3, 6, and 28)

[IL89] Russell Impagliazzo and Michael Luby. One-way functions are essential for complexity based cryptography (extended abstract). In 30th Annual Symposium on Foundations of 
Computer Science, pages 230-235, Research Triangle Park, North Carolina, October 30 November 1, 1989. IEEE Computer Society Press. (Cited on page 35])

[KPTZ13] Aggelos Kiayias, Stavros Papadopoulos, Nikos Triandopoulos, and Thomas Zacharias. Delegatable pseudorandom functions and applications. In Ahmad-Reza Sadeghi, Virgil D. Gligor, and Moti Yung, editors, ACM CCS 13: 20th Conference on Computer and Communications Security, pages 669-684, Berlin, Germany, November 4-8, 2013. ACM Press. (Cited on pages 6 15 , and 35 )

[LPS04] Ben Lynn, Manoj Prabhakaran, and Amit Sahai. Positive results and techniques for obfuscation. In Christian Cachin and Jan Camenisch, editors, Advances in Cryptology EUROCRYPT 2004, volume 3027 of Lecture Notes in Computer Science, pages 20-39, Interlaken, Switzerland, May 2-6, 2004. Springer, Berlin, Germany. (Cited on page 13 )

[MH14a] Takahiro Matsuda and Goichiro Hanaoka. Chosen ciphertext security via point obfuscation. In Yehuda Lindell, editor, TCC 2014: 11th Theory of Cryptography Conference, volume 8349 of Lecture Notes in Computer Science, pages 95-120, San Diego, CA, USA, February 24-26, 2014. Springer, Berlin, Germany. (Cited on pages 7 8, 13,28 , and 35)

[MH14b] Takahiro Matsuda and Goichiro Hanaoka. Chosen ciphertext security via UCE. In Hugo Krawczyk, editor, PKC 2014: 17th International Conference on Theory and Practice of Public Key Cryptography, volume 8383 of Lecture Notes in Computer Science, pages 56-76, Buenos Aires, Argentina, March 26-28, 2014. Springer, Berlin, Germany. (Cited on page 6)

[Mit14] Arno Mittelbach. Salvaging indifferentiability in a multi-stage setting. In Phong Q. Nguyen and Elisabeth Oswald, editors, Advances in Cryptology - EUROCRYPT 2014, volume 8441 of Lecture Notes in Computer Science, pages 603-621, Copenhagen, Denmark, May 11-15, 2014. Springer, Berlin, Germany. (Cited on page 6)

[PST14] Rafael Pass, Karn Seth, and Sidharth Telang. Indistinguishability obfuscation from semantically-secure multilinear encodings. In Juan A. Garay and Rosario Gennaro, editors, Advances in Cryptology - CRYPTO 2014, Part I, volume 8616 of Lecture Notes in Computer Science, pages 500-517, Santa Barbara, CA, USA, August 17-21, 2014. Springer, Berlin, Germany. (Cited on page 34)

[RS10] Alon Rosen and Gil Segev. Chosen-ciphertext security via correlated products. SIAM Journal on Computing, 39(7):3058-3088, 2010. (Cited on page 9)

[SW14] Amit Sahai and Brent Waters. How to use indistinguishability obfuscation: deniable encryption, and more. In David B. Shmoys, editor, 46th Annual ACM Symposium on Theory of Computing, pages 475-484, New York, NY, USA, May 31 - June 3, 2014. ACM Press. (Cited on pages 3, 4, 6, 15, and 28,)

[Wee05] Hoeteck Wee. On obfuscating point functions. In Harold N. Gabow and Ronald Fagin, editors, 37th Annual ACM Symposium on Theory of Computing, pages 523-532, Baltimore, Maryland, USA, May 22-24, 2005. ACM Press. (Cited on page 34.)

[Wic13] Daniel Wichs. Barriers in cryptography with weak, correlated and leaky sources. In Robert D. Kleinberg, editor, ITCS 2013: 4th Innovations in Theoretical Computer Science, pages 111-126, Berkeley, CA, USA, January 9-12, 2013. Association for Computing Machinery. (Cited on pages 3 and 4) 


\section{A Constructions and Candidates for Obfuscation Schemes}

In the following we present existing candidates for indistinguishability obfuscation (which also yield restricted forms of differing-inputs obfuscation with a beautiful result of Boyle, Chung and Pass [BCP14]) and point obfuscation schemes with auxiliary input (AIPOs).

INDISTINGUISHABILITY OBFUSCATION. In a breakthrough paper, Garg et al. [GGH ${ }^{+} 13$ present a candidate construction for indistinguishability obfuscation. Their candidate is based on an intractability assumption related to multi-linear maps and their construction yields an indistinguishability obfuscator for all circuits in $\mathcal{N C}^{1}$. They go on to show that, if additionally assuming a perfectly correct leveled fully homomorphic encryption scheme and a perfectly sound non-interactive witness-indistinguishable proof system that their obfuscator can be bootstrapped to yield an indistinguishability obfuscator for all circuits in $\mathcal{P} /$ poly. In recent works, Brakerski and Rothblum [BR14] and Barak et al. [BGK ${ }^{+} 14$ ] have further simplified the construction and showed that it is secure against all generic multi-linear attacks. A result by Ananth et al. AGIS14 yields a more efficient construction. Complementary, Pass, Seth and Telang [PST14] show how to base an adapted construction on a novel assumption they call semantically-secure multilinear encodings. In a very recent work Gentry et al. GLSW14 show that iO can be based on instance-independent assumptions and give a construction based on the Multilinear Subgroup Elimination Assumption.

Candidates for point obfuscation with auxiliary inPut (AIPO). The study of point function obfuscation started with Canetti [Can97] who gives a construction that satisfies Definition 2.7 under a strong variant of the DDH assumption. We here present the construction in the formulation of [BP12] and then present the assumption it is based on.

Construction A.1 (AIPO obfuscator due to [Can97]). Let $\mathcal{G}:=\left\{\mathbb{G}_{\lambda}\right\}_{\lambda \in \mathbb{N}}$ be a group ensemble, where each $\mathbb{G}_{\lambda}$ is a group of prime order $p_{\lambda} \in\left(2^{\lambda-1}, 2^{\lambda}\right)$. We define an obfuscator AIPO for points in the domain $\mathbb{Z}_{p_{\lambda}}$ as follows: $p_{x} \stackrel{\text { IIPO }}{\mapsto} C\left(r, r^{x}\right)$, where $r \leftarrow \$ \mathbb{G}_{\lambda}$ is a random generator of $\mathbb{G}_{\lambda}$, and $C\left(r, r^{x}\right)$ is a circuit which on input $i$, checks whether $r^{x}=r^{i}$.

Assumption A.2 ([Can97, BP12]). There exists an ensemble of prime order groups $\mathcal{G}:=\left\{\mathbb{G}_{\lambda}\right\}_{\lambda \in \mathbb{N}}$ such that for any unpredictable distribution $\mathcal{D}=\left\{D_{\lambda}=\left(Z_{\lambda}, Y_{\lambda}\right)\right\}_{\lambda \in \mathbb{N}}$ with support $\{0,1\}^{\text {poly }(\lambda)} \times \mathbb{Z}_{p_{\lambda}}$, it holds that for all PPT algorithms $\mathcal{A}$ there exists a negligible function negl such that

$$
\left|\operatorname{Pr}_{r \leftrightarrow \mathbb{G}_{\lambda}, \leftrightarrow(z, x) \leftrightarrow D_{\lambda}}\left[\mathcal{A}\left(z, r, r^{x}\right)=1\right]-\operatorname{Pr}_{r \leftrightarrow \mathbb{G}_{\lambda}, z \leftrightarrow Z_{\lambda}, u \leftrightarrow \mathbb{Z}_{p_{\lambda}}}\left[\mathcal{A}\left(z, r, r^{u}\right)=1\right]\right| \leq \operatorname{neg}(\lambda)
$$

A second candidate construction for AIPO is due to Bitansky and Paneth BP12] who adapt a point obfuscation construction by Wee [Wee05] to allow for auxiliary input. Their construction is based on an assumption on the existence of strong pseudorandom permutations. Let us recall the underlying assumption (which generalizes the original assumption due to Wee [Wee05]) before recalling the construction.

Assumption A.3 ([BP12]). There exists an ensemble of permutation families $\mathcal{F}=\left\{\mathcal{F}_{\lambda}=\{f\}\right\}$ such that for any unpredictable distribution ensemble $\mathcal{D}=\left\{D_{\lambda}=\left(Z_{\lambda}, Y_{\lambda}\right)\right\}_{\lambda \in \mathbb{N}}$, the following two distribution ensembles are also unpredictable:

- $\left(\left(Z_{\lambda}, f\left(Y_{\lambda}\right), f\right) ; Y_{\lambda}\right)$

- $\left(\left(Z_{\lambda}, f\right) ; f\left(Y_{\lambda}\right)\right)$, 
where in both $f \leftarrow \$ \mathcal{F}_{\lambda}$ (independently of $D_{\lambda}$ ).

Based on Assumption A.3, Bitansky and Paneth show that the following construction yields an AIPO obfuscator satisfying Definition 2.7 BP12].

Construction A.4 ([BP12]). Let $\mathcal{F}$ be a family of permutations as given by Assumption A.3. AIPO obfuscator AIPO works as follows: given a point $y \in\{0,1\}^{\lambda}$, AIPO samples $3 \lambda$ permutations $\left\{f_{i}\right\}_{i \in[3 \lambda]}$ from $\mathcal{F}_{\lambda}$ and $3 \lambda$ strings $\left\{r_{i}\right\}_{i \in[3 \lambda]}$ from $\{0,1\}^{\lambda}$. For every $i \in[3 \lambda]$, let $f^{i}:=f_{i} \circ f_{i-1} \circ \ldots \circ f_{1}$ (where $\circ$ denotes composition). Obfuscator AIPO outputs a circuit $C_{y}$ that has hardcoded into it the randomness of AIPO, $\left\{f_{i}, r_{i}\right\}_{i \in[3 \lambda]}$ and the bits $\left\{b_{i}:=\left\langle r_{i}, f^{i}(y)\right\rangle\right\}_{i \in[3 \lambda]}$, where $\langle.,$.$\rangle denotes the inner product over$ $\mathbb{G F}_{2}$. Circuit $C_{y}$ outputs 1 on a point $x$ if for all $i \in[3 \lambda]: b_{i}=\left\langle r_{i}, f^{i}(x)\right\rangle$; and 0 otherwise.

Statistical AIPO. Bitansky and Canetti show that the point obfuscation scheme of Canetti Can97. is a $t$-composable VGB point obfuscator and that $t$-composable VGB point obfuscation implies obfuscation in the presence of auxiliary information (VGB-AI). Matsuda and Hanoka [MH14a] relate the notions of VGB point obfuscators (resp. VGB-AI point obfuscators) and AIPO and show that composable VGB-AI point obfuscators imply the existence of composable AIPO with respect to statistically unpredictable distributions.

\section{B AIPO Implies One-way Functions}

In this section, we show that, like most cryptographic primitives, AIPO implies one-way functions. As one-way functions imply PRGs [HILL99] and as PRGs imply puncturable PRFs [BW13, BGI14, KPTZ13], that suffices to prove that AIPO implies puncturable PRFs.

Lemma B.1 (AIPO implies one-way functions). Point Function Obfuscation (that is secure under auxiliary inputs) implies puncturable PRFs.

Proof. Two distributions that are statistically close and computationally far imply a distributional oneway function [Gol90, and a distributionally one-way function implies a standard one-way function [IL89. The security property of AIPO implies that the obfuscation of $p_{x}$ for a random $x$, where $x_{1}=0$ (i.e., the first bit of $x$ is 0 ) is indistinguishable from the obfucation of $p_{u}$ for a random $u$. Hence, we have two computationally indistinguishable distributions. Let us argue that they are statistically far. With probability $\frac{1}{2}$, the first bit of $u$ does not equal 0 and hence, the obfuscation of $u$ is outside of the support of the distributions over $p_{x}$. Hence, the two random variables have statistical distance at least $\frac{1}{2}$ which concludes the proof.

\section{Proof of Theorem 3.5}

The main differences compared to the proof of Theorem 3.3 is that we now puncture on $q$ many points and also construct $q$ many point obfuscations and hence, in the analogue to Claim 3.4 we need to use composable point obfuscations. We now describe the five games and then give the proof of the analogue to Claim 3.4. We present the pseudocode for the games in Figure 9. Note that we assume, without loss of generality, that the source always makes $q$ distinct queries. Further note the notation we have chosen to store the queries: we use two lists $X^{*}, Y^{*}$ to store queries $x$ and corresponding answer $y$. In order to emulate that in strong unpredictability the query answers are not given in an ordered list to the predictor but as an unordered set we fill the lists $X^{*}$ and $Y^{*}$ in a random order using the helper set Indexes (see Figure 9). 
Game $_{1}$ : The first game is the original $\operatorname{UCE}\left[\mathcal{S}^{\text {s-sup }} \cap \mathcal{S}^{q \text {-query }}\right]$-game. Here, the hash key hk is an obfuscation of the circuit $C_{1}[k](x):=G$. Eval $(k, x)$ where $k$ is a key for the puncturable PRF.

Game 2 : Let $x_{1}^{*}, \ldots, x_{q}^{*}$ denote the $q$ queries that the source $\mathrm{S}$ makes to its HAsH oracle and let

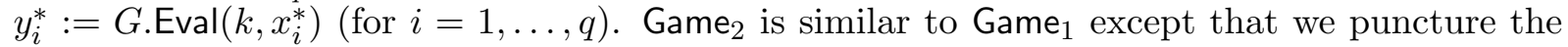
PRF on $x_{1}^{*}$ to $x_{q}^{*}$. Namely, the hash key hk does not consist of an obfuscation of $C_{1}[k]$ anymore, but rather of an obfuscation of the circuit $C_{2}\left[k^{*}, p_{1}, \ldots, p_{q}, y_{1}^{*}, \ldots, y_{q}^{*}\right]$. The circuits $C_{1}[k]$ and $C_{2}\left[k^{*}, p_{1}, \ldots, p_{q}, y_{1}^{*}, \ldots, y_{q}^{*}\right]$ are functionally equivalent. However, instead of the normal PRF key, $C_{2}$ uses a punctured PRF key $k^{*}$ which is punctured at values $x_{i}^{*}$ (for $i=1, \ldots, q$ ). Here, $p_{i}$ is computed as the point obfuscation of the point function $p_{x_{i}^{*}}$. On input a value $x$, circuit $C_{2}\left[k^{*}, p_{1}, \ldots, p_{q}, y_{1}^{*}, \ldots, y_{q}^{*}\right]$ checks whether there exists $i \in\{1, \ldots, q\}$ such that $p_{i}(x)=\perp$. If so, it returns $y_{i}^{*}$, otherwise it outputs $G$. Eval $\left(k^{*}, x\right)$.

Game 3 : The game is equivalent to Game 2 except that oracle HASH now samples $y_{i}^{*}$ uniformly at random instead of invoking $G$. Eval $(k,$.$) . Note that C_{2}\left[k^{*}, p_{1}, \ldots, p_{q}, y_{1}^{*}, \ldots, y_{q}^{*}\right]$ is parametrized by $y_{i}^{*}$.

Game 4 : The game is equivalent to the previous game except that we now use an obfuscation of circuit $C_{3}\left[k, p_{1}, \ldots, p_{q}, y_{1}^{*}, \ldots, y_{q}^{*}\right]$ as hash key hk. Circuit $C_{3}\left[k, p_{1}, \ldots, p_{q}, y_{1}^{*}, \ldots, y_{q}^{*}\right]$ is identical to circuit $C_{2}\left[k^{*}, p_{1}, \ldots, p_{q}, y_{1}^{*}, \ldots, y_{q}^{*}\right]$, except that it uses the original PRF key $k$ instead of the punctured key $k^{*}$. Note that circuits $C_{3}\left[k, p_{1}, \ldots, p_{q}, y_{1}^{*}, \ldots, y_{q}^{*}\right]$ and $C_{2}\left[k^{*}, p_{1}, \ldots, p_{q}, y_{1}^{*}, \ldots, y_{q}^{*}\right]$ have identical input-output behaviour.

Game 5 : The game is equivalent to the previous game except that now an obfuscation of circuit $C_{4}[k]$ is used as hash key hk. Circuit $C_{4}[k]$ is our original circuit again, that is, $C_{4}[k](\cdot):=G$. Eval $(k, \cdot)$. Game $_{5}$ is our intended target. It is the UCE-security game for our construction in the random oracle world (that is, oracle HASH implements a random oracle).

It remains to give the analogue of Claim 3.4

Claim C.1. If AIPO is a q-composable AIPO obfuscator for statistically hard-to-invert auxiliary information, then the family of circuit pairs

$$
\left(C_{3}\left[k, p_{1}, \ldots, p_{q}, y_{1}^{*}, \ldots, y_{q}^{*}\right], C_{4}[k], \mathrm{Sam}\right)
$$

is differing-inputs.

Proof. Assume there exists an adversary (i.e., an extractor) Ext against the differing-inputs of the above circuit family which receives as input $\left(C_{3}\left[k, p_{1}, \ldots, p_{q}, y_{1}^{*}, \ldots, y_{q}^{*}\right], C_{4}[k], L\right)$ and outputs a value $\tau$ such that $C_{3}\left[k, p_{1}, \ldots, p_{q}, y_{1}^{*}, \ldots, y_{q}^{*}\right](\tau) \neq C_{4}[k](\tau)$. Then, $p_{i}(\tau)=1$ for some $i \in\{1, \ldots, q\}$ and thus, intuitively, Ext breaks the security property of the point obfuscation scheme. Let us now make this intuition formal.

We construct adversary $\left(\mathcal{B}_{1}, \mathcal{B}_{2}\right)$ where $\mathcal{B}_{1}$ describes a statistically unpredictable distribution. On input the security parameter, $\mathcal{B}_{1}$ runs source $S\left(1^{\lambda}\right)$. Without loss of generality we assume that the source's queries are distinct. Adversray $\mathcal{B}_{1}$ answers the $q$ many distinct HAsH queries $x_{i}^{*}$ each with a uniformly random value $y_{i}^{*}$ and then receives leakage $L$ from $\mathrm{S}$. Adversary $\mathcal{B}_{1}$ then draws a random string $r$ and index $j \leftarrow \$\{1, \ldots, q\}$. It computes $b:=\left\langle r, x_{j}^{*}\right\rangle$ and chooses a random permutation perm $q$ on the set $[q]=\{1, \ldots, q\}$. That is $\forall i \in[q]: \operatorname{perm}_{q}(i) \in[q]$ and $\forall i, j \in[q]: \operatorname{perm}_{q}(i) \neq \operatorname{perm}_{q}(j)$. Finally adversary $\mathcal{B}_{1}$ outputs $\left(\left(x_{\text {perm }_{q}(1)}^{*}, \ldots, x_{\text {perm }_{q}(q)}^{*}\right),\left(b, r, \operatorname{perm}_{q}(j), y_{\text {perm }_{q}(1)}^{*}, \ldots, y_{\text {perm }_{q}(q)}^{*}, L\right)\right)$. That is, it permutes the queries $x_{i}^{*}$ and corresponding images $y_{i}^{*}$ while keeping index $j$ intact. Note that the 


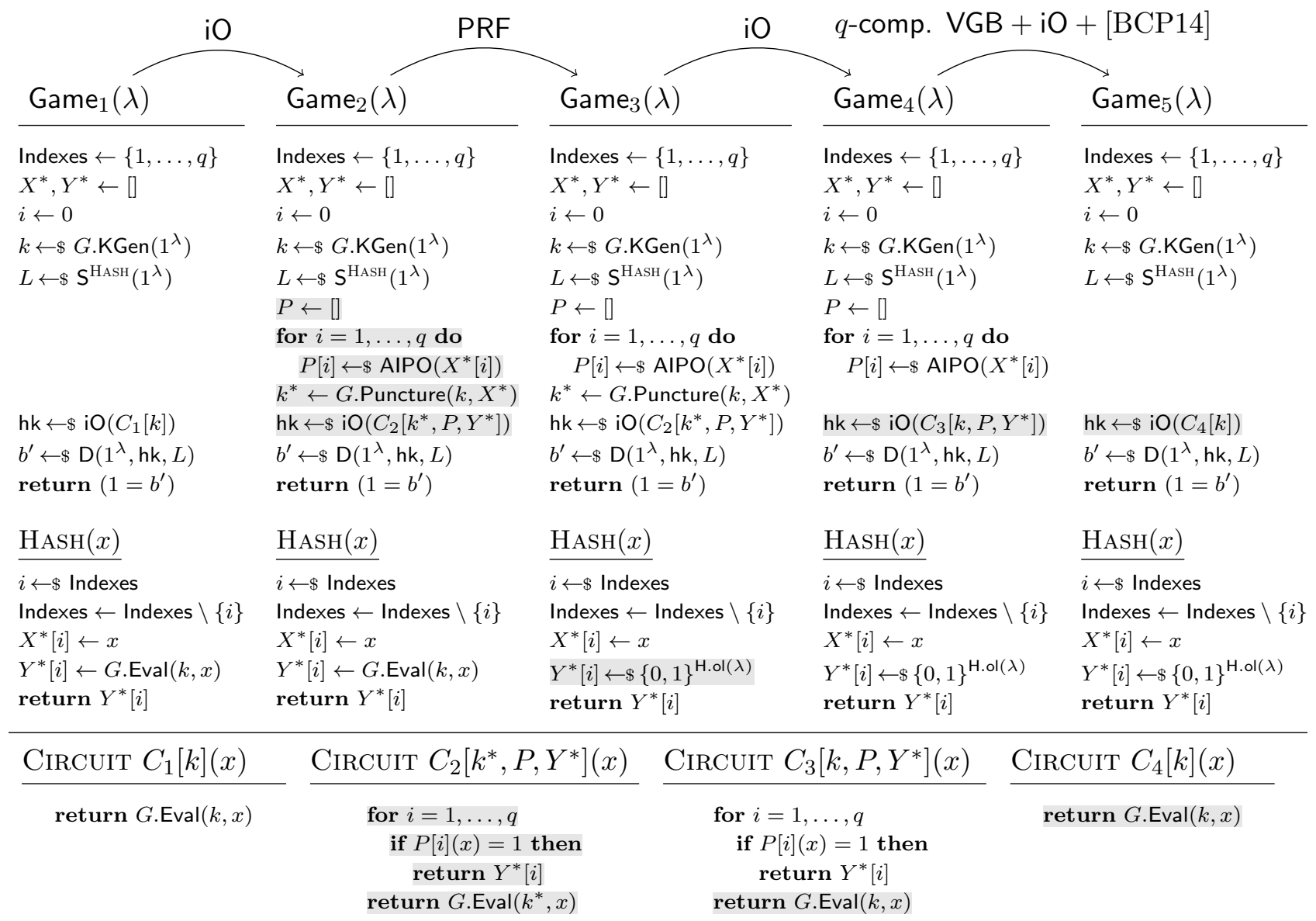

Figure 9: The games used in the proof of Theorem 3.5 on the top and the corresponding circuits on the bottom. Note that we use lists $X^{*}, Y^{*}$ and $P$ to store queries, answers and point functions. Further note that the lists $X^{*}$ and $Y^{*}$ are filled in a random order emulating a set (with the exception that collisions are kept).

usage of the random permutation emulates the random assignment of indexes in games Game 3 and $\mathrm{Game}_{4}$ (see Figure 9).

Adversary $\mathcal{B}_{2}$ gets as input the security parameter, the auxiliary input $\left(b, r, j, y_{1}^{*}, \ldots, y_{q}^{*}, L\right)$, as well as $q$ obfuscations $p_{1}, \ldots, p_{q}$ which are either honest obfuscations of point functions $p_{x_{i}^{*}}$ (for $i=1, \ldots, q)$ or of q uniformly random points. It samples a random key $k \leftarrow \$ G$. KGen $(\lambda)$ and constructs circuits $C_{3}\left[k, p_{1}, \ldots, p_{q}, y_{1}^{*}, \ldots, y_{q}^{*}\right]$ and $C_{4}[k]$. It then calls the extractor Ext on input $\left(C_{3}\left[k, p_{1}, \ldots, p_{q}, y_{1}^{*}, \ldots, y_{q}^{*}\right], C_{4}[k], L\right)$ to receive a value $\tau$. If Ext outputs $\tau=\perp$, then $\mathcal{B}_{2}$ flips a bit and returns the outcome of the bitflip. Else, if $\tau$ is such that $C_{3}\left[k, p_{1}, \ldots, p_{q}, y_{1}^{*}, \ldots, y_{q}^{*}\right](\tau) \neq C_{4}[k](\tau)$ and $p_{j}(\tau)=1$, then $\mathcal{B}_{2}$ outputs 1 if $\langle r, \tau\rangle$ equals $b$ and 0 otherwise. Else, if $p_{j}(\tau)=\perp$, then $\mathcal{B}_{2}$ also flips a bit and returns the outcome of the bitflip.

If the obfuscations where chosen honestly with respect to the target points $x_{1}^{*}, \ldots, x_{q}^{*}$, then circuits $C_{3}\left[k, p_{1}, \ldots, p_{q}, y_{1}^{*}, \ldots, y_{q}^{*}\right]$ and $C_{4}[k]$ differ on input $\tau$ if and only if $\tau=x_{i}^{*}$ for some $i \in\{1, \ldots, q\}$. Hence, if the differing-inputs adversary Ext outputs $\tau$, then $\tau=x_{i}^{*}$ for some $i \in\{1, \ldots, q\}$ and, thus, with probability $1 / q$ value $\tau$ is equal to $x_{j}^{*}$ and, hence, $\mathcal{B}_{2}$ will output 1 . If, on the other hand, the obfuscations $p_{i}$ are of random points $u_{i}$, then the circuits $C_{3}\left[k e y, p_{1}, \ldots, p_{q}, y_{1}^{*}, \ldots, y_{q}^{*}\right]$ and $C_{4}[k]$ differ on input $\tau$ if and only if $\tau=u_{i}$ for some $i \in\{1, \ldots, q\}$. Hence, if the differing-inputs adversary Ext outputs $\tau$ and $\tau=u_{j}$ then $\mathcal{B}$ will only output 1 with probability $\frac{1}{2}\left(\operatorname{since} \operatorname{Pr}\left[\left\langle u_{j}, r\right\rangle=b\right]=\frac{1}{2}\right)$. The formal analysis is equivalent to the one for Claim 3.4 with an additional loss of factor $\frac{1}{q}$ for guessing 
the right index.

To finish the proof of Claim C.1. we need to argue that $\mathcal{B}_{1}$ implements a statistically unpredictable distribution. By assumption, the source $\mathrm{S}$ is strongly, statistically unpredictable (i.e., $\mathrm{S} \in \mathcal{S}^{\text {s-sup }}$ ) and hence leakage $L$ hides the query points even in the presence of a set containing the HAsH answers. As all $y_{i}$ in the simulation are chosen uniformly at random the probability of a collision is negligible. (Note that strong unpredictability is only guaranteed in the presence of the set of answers of the oracle, and if a collision occurs $\mathcal{B}_{1}$ violates this condition.) Additionally, note that $B_{1}$ outputs values $y_{i}^{*}$ not in their correct order but in a random order perfectly mimicking the requirements of a strongly unpredictable source. Thus, to see that $\mathcal{B}_{1}$ defines an unpredictable distribution, we need to argue that $x_{i}^{*}$ remain unpredictable if additionally given a single bit of $x_{j}^{*}$ and an index $j$. But a single bit and index can be guessed with probability $\frac{1}{2 q}$. Hence, $\left(\mathcal{B}_{1}, \mathcal{B}_{2}\right)$ breaks the security of the AIPO obfuscation, which concludes the proof of Claim C.1. 